\title{
Joint Discussion 3 Solar active regions and 3D magnetic structure
}

\author{
Debi Prasad Choudhary ${ }^{1}$ and Michal Sobotka ${ }^{2}$ (eds.) \\ ${ }^{1}$ Department of Physics and Astronomy, California State University Northridge, \\ 18111 Nordhoff Street, Northridge, CA, 19330, USA \\ email: debiprasad.choudhary@csun.edu \\ ${ }^{2}$ Astronomical Institute, Academy of Sciences of the Czech Republic, \\ Fričova 298, 25165 Ondřejov, Czech Republic \\ email: msobotka@asu.cas.cz
}

\begin{abstract}
Keeping in view of the modern powerful observing tools, among others Hinode (formerly SOLAR-B), STEREO and Frequency-Agile Solar Radiotelescope, and sophisticated modelling techniques, Joint Discussion 3 during the IAU General Assembly 2006 focused on the properties of magnetic field of solar active regions starting in deep interior of the Sun, from where they buoyantly rise to the coronal heights where the site of most explosive events are located. Intimately related with the active regions, the origin and evolution of the magnetic field of quiet Sun, the large scale chromospheric structures were also the focal point of the Joint Discussion. The theoretical modelling of the generation and dynamics of magnetic field in solar convective zone show that the interaction of the magnetic field with the Coriolis force and helical turbulent convection results in the tilts and twists in the emerging flux. In the photosphere, some of these fluxes appear in sunspots with field strengths up to about $6100 \mathrm{G}$. Spectro-polarimetric measurements reveal that the line of sight velocities and magnetic field of these locations are found to be uncombed and depend on depth in the atmosphere and exhibit gradients or discontinuities. The inclined magnetic fields beyond penumbra appear as moving magnetic features that do not rise above upper photospheric heights. As the flux rises, the solar chromosphere is the most immediate and intermediary layer where competitive magnetic forces begin to dominate their thermodynamic counterparts. The magnetic field at these heights is now measured using several diagnostic lines such as Ca II $854.2 \mathrm{~nm}$, H I $656.3 \mathrm{~nm}$, and He I $1083.0 \mathrm{~nm}$. The radio observations show that the coronal magnetic field of post flare loops are of the order of $30 \mathrm{G}$, which might represent the force-free magnetic state of active region in the corona. The temperatures at these coronal heights, derived from the line widths, are in the range from 2.4 to 3.7 million degree. The same line profile measurements indicate the existence of asymmetric flows in the corona. The theoretical extrapolation of photospheric field into coronal heights and their comparison with the observations show that there exists a complex topology with separatrices associated to coronal null points. The interaction of these structures often lead to flares and coronal mass ejections. The current MHD modelling of active region field shows that for coronal mass ejection both local active region magnetic field and global magnetic field due to the surrounding magnetic flux are important. Here, we present an extended summary of the papers presented in Joint Discussion 03 and open questions related to the solar magnetic field that are likely to be the prime issue with the modern observing facilities such as Hinode and STEREO missions.
\end{abstract}

Keywords. Solar activity, sunspots, magnetic field, corona, chromosphere, flare, radio observations, CME, magnetic field extrapolation 


\section{Introduction}

The magnetic field of the Sun is responsible for most of its visible dynamic features, including the most energetic events that can affect the near earth space environment, producing space weather. The solar magnetic field is generated below the visible layer (the photosphere) and erupts into the solar atmosphere. The cross-section of the erupting field structure at the photosphere is observed as an active region, above which there exists a complex three-dimensional magnetic "dome". Many fundamental physical processes take place in and above active regions that govern the dynamics of the hot, magnetized plasma manifesting in the observed features. In order to understand these processes, a detailed knowledge of the origin and dynamics of magnetic field is essential. In the recent past there have been spectacular advances in various topics related to this field such as: magnetic helicity, temporal evolution of magnetic field creating large-scale structure; thermal and magnetic instabilities leading to fine-scale structure; wave dissipation and reconnection providing coronal heating; instability and non-equilibrium states leading to eruptions. The future observations in infrared wavelength and from space platforms combined with the sophisticated computer modeling are expected to make equally impressive advances.

The photospheric vector magnetic field of solar active regions has been measured on a synoptic basis for the last 30 years. From these, the 3D magnetic field is derived using numerical models. The spatial resolution of the measurements has improved steadily, and the models have been able to incorporate some departures from the force-free field approximation. Several of there models are now capable of reproducing the observed sheared coronal magnetic features.

Until now the field in the chromosphere and corona has been largely derived by extrapolating the photospheric measurements. Recently, the actual measurements of these fields have been carried out using both the Zeeman and Hanle effects. In the last few years, several exploratory measurements of magnetic fields in spectral lines originating at chromospheric and coronal heights have shown promising results. The technological advances in the field of detectors and polarizing optics make it possible to design and fabricate vector magnetographs that can be used to measure vector magnetic fields simultaneously in several layers above the photosphere. The longitudinal chromospheric field measurements using the Zeeman effect have been carried out on a regular basis at NSO/KP, Hawaii and Huairou in China and are being planned at NASA/MSFC and San Fernando Observatory. At the same time the Hanle effect has emerged as a new and powerful diagnostic tool. The Hanle effect is useful both for measuring weak horizontal magnetic fields in the solar chromosphere, and for exploring the sub-resolution tangled or turbulent magnetic fields. The turbulent magnetic field cannot be seen by the Zeeman effect, but has been found to carry a vast amount of "hidden" magnetic flux. The recently uncovered wealth of polarization phenomena throughout the "Second Solar Spectrum", formed by coherent scattering processes, has an exciting and entirely novel diagnostic potential.

The other aspect of solar magnetic field is inverting the observed polarization measurements in deriving the magnetic field. There is a considerable progress both in terms of atomic and molecular physics and radiative transfer analysis in this crucial topic. On the other hand, helioseismic analysis is beginning to provide interesting results in the sub-photospheric structure and flows in active regions. These results can be used to derive sub-photospheric magnetic fields in active regions and may in the near future also help us in refining models about how active regions emerge.

There are many new instruments that are now under development and poised to produce impressive results in near future. The solar optical space telescope with $50 \mathrm{~cm}$ 
aperture and its back-end instrumentation on Hinode (Solar-B), successfully launched in September 2006, is poised to revolutionize our understanding the fine scale magnetic structure on the Sun. Other space initiatives such as STEREO will provide us the stereoscopic three dimensional vision of solar magnetic structures and their dynamics. Many ground-based instruments such as GREGOR, new 1-m SST at La Palma and NSO/SP adaptive optics are expected to yield new results in this field. There are other equally impressive instruments such as the chromospheric magnetograph at BBSO, Hawaii, IR spectropolarimeter at San Fernando Observatory of California State University Northridge and Norikura Solar Observatory, Japan, that are making important contribution in terms of magnetic field measurements. On the other hand radio emission is sensitive to magnetic fields throughout the solar atmosphere, and there is a long history of such measurements. A new radio facility, the Frequency-Agile Solar Radiotelescope (FASR) is now being designed with a main goal of routinely measuring coronal magnetic fields at high spatial resolution. In this symposium we shall focus to the measurement of solar magnetic field in different wavelengths and different techniques.

With this scientific rationale, the Joint Discussion 3 (JD03) was organized to summarize the progress in this field and explore the possible outstanding problems that can be addressed with the future instruments. The one and half day discussion consisted of 11 invited talks, 15 oral and 65 poster presentation demonstrating the overwhelming response. JD03 dealt with topics related to magnetic field in the solar convective zone, the observation and inversion techniques for photospheric, chromospheric and coronal magnetic field and small-scale structures. The role and properties of magnetic structure in eruptive processes leading to flares, filament eruptions and Coronal Mass Ejections were also the subject of JD03. This summary is divided into sections dealing with different parts of the Sun.

\section{Magnetic field in the convection zone}

The sunspots are the cross sections of the magnetic structures that protrude into the solar atmosphere through the photosphere. The kinematic dynamo model among others was reviewed by Dibyendu Nandy. He showed that the toroidal field is generated by strong differential rotation in the tachocline. The buoyant eruptions bring the flux to photospheric layers. The decay of tilted active regions produce the poloidal field. The entire evolution of the solar magnetic field is governed by $\alpha$ - and $\omega$-effects, meridional circulation and magnetic buoyancy as shown in Fig. 1. Using observed large-scale flows it is now possible to reproduce the observed large-scale magnetic field evolution reasonably well. While the meridional circulation transports poloidal and toroidal flux, the diffusion transports and also destroys them. The three possible sources of $\alpha$-effect that govern the surface flux dynamics are (1) Coriolis force during flux tube rise in convection zone, (2) diffusion of tilted dipole fields at surface, or (3) differential rotation in tachocline. The flux transport mediated time-delay dynamics introduces a memory mechanism in the Sun that may be used for predictive purposes. The question remains if the local and global dynamos are linked? The tilt and twist of the flux are generated by the Coriolis force and helical convection. The Coriolis force in the northern hemisphere would produce negative kinetic helicity that will distort the axis of rising flux tubes in a right handed sense (positive writhe), therefore negative tilt, and vice-versa in the south will generate a negative twist in the southern hemisphere. Analysis of twist distribution and its dependence on latitude suggests that the Solar Convection Zone (SCZ) turbulence is isotropic in latitude. The Active Region (AR) twist dependence on flux indicates that larger flux tubes are less affected by turbulent buffeting and hence pick up less twist 


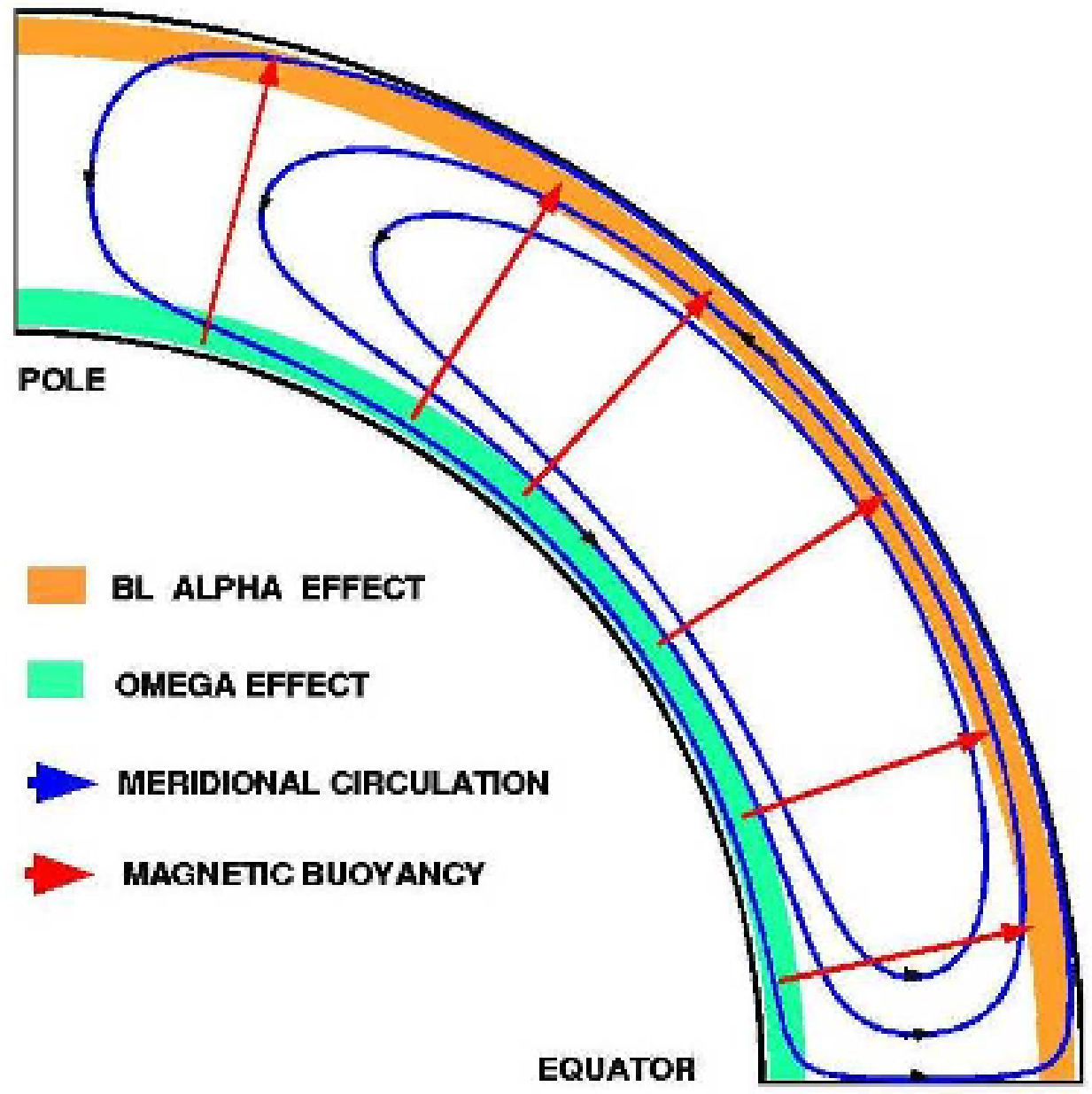

Figure 1. The sites of $\alpha$ and $\omega$ effects, buoyant force and meridional flow.

and scatter. Analysis of twist and tilt relationship supports a scenario where flux tubes at the base of the SCZ begin their outward journey with a wide range of initial twist, some with high enough twist to make them kink unstable. This may suggest that some physical process at the base of the SCZ, e.g., the dynamo or the process of flux tube formation, creates or imparts this initial twist.

The results of the numerical simulations flux ropes in the magnetic SCZ by Soren B.F. Dorch showed how twisted magnetic flux ropes interact with a magnetized model envelope. Buoyant magnetic flux ropes have been extensively studied through numerical simulations, cf. the review in Fan (2004). However, so far studies of the interaction of flux ropes with poloidal fields have solely dealt with flux ropes that emerge into the solar corona, e.g., Archontis et al. (2004), i.e., not with a magnetic SCZ. The question 


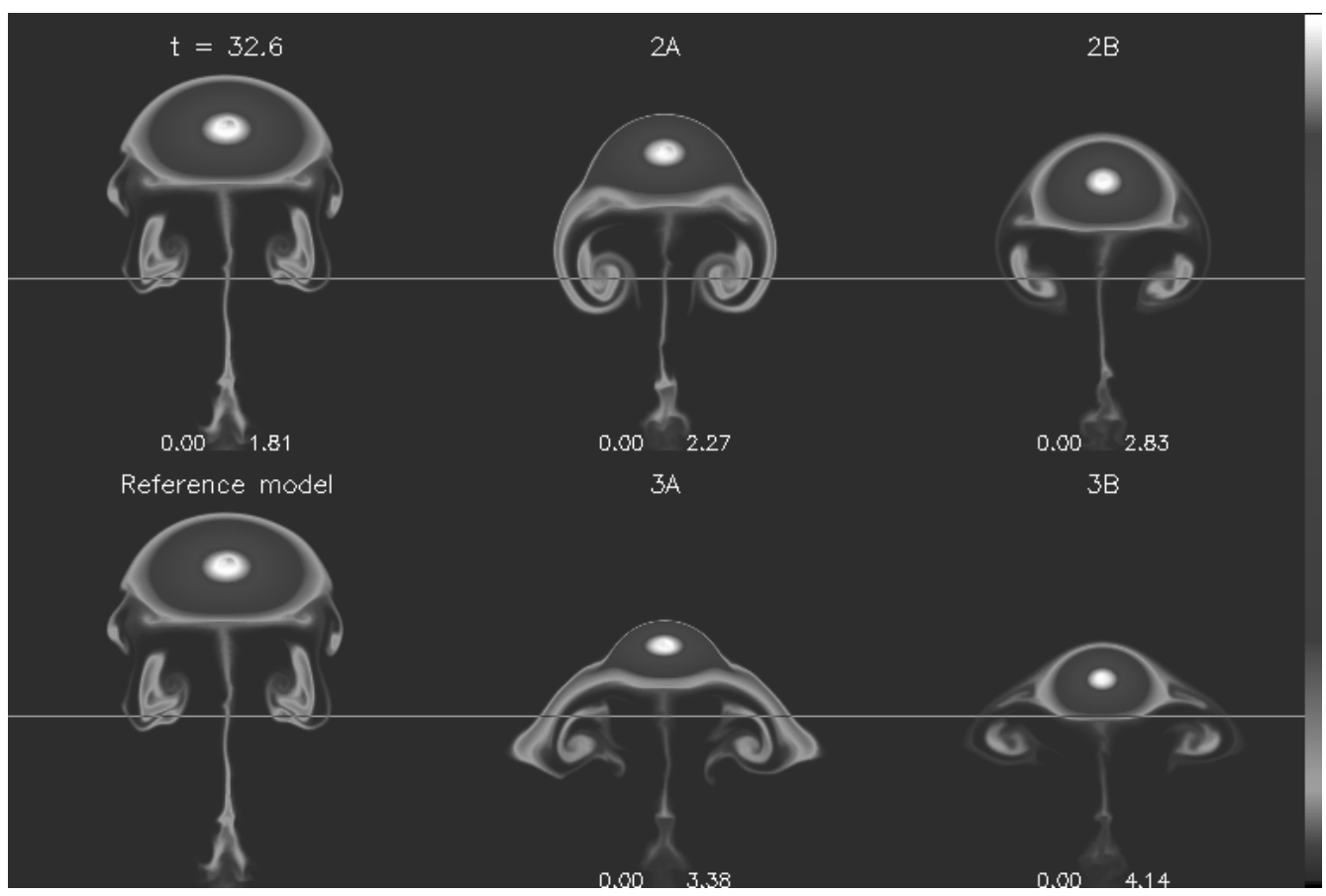

Figure 2. Grey-scale panel showing snapshots of parallel magnetic field $B_{z}$ at $t \simeq 32.6$ time units for models with moderately magnetized envelopes. Minimum (zero) and maximum magnetic field strengths are stated for every snapshot (in units of $354 \mathrm{kG}$ ). Vertical dimension corresponds to $x$ in the simulations. Shown are a reference model (shown twice), and in the upper row two models with initial $B_{\mathrm{SCZ}} / B_{\phi}=18$ (but oppositely twisted) and in the lower row two models with initial $B_{\mathrm{SCZ}} / B_{\phi}=9$ (and oppositely twisted). Also shown are horizontal lines indicating the initial position of the magnetized layer.

addressed here is how the polarity and strength of the field in a magnetized convection zone may affect the rise and evolution of twisted flux ropes. When the predominantly toroidal buoyant flux ropes rise through the SCZ, they encounter a poloidal magnetic field that has a component perpendicular to the ropes' axes. However, since the flux ropes are twisted, the transversal field components may be either parallel or anti-parallel to the average magnetic field in the SCZ. Using the MHD code by Galsgaard \& Nordlund (1997) and others, Dorch performed several 2.5-dimensional numerical simulations of buoyant twisted flux ropes rising into a uniform magnetic field, cf. Dorch (2007). Some of the results are illustrated in Fig. 2 that shows 2-dimensional cross sections of the flux ropes (the parallel field strength $B_{\mathrm{z}}$ ). There are both quantitative and qualitative differences depending on the ratio of the SCZ magnetic field to the rope's twisted field component, i.e., $B_{\mathrm{SCZ}} / B_{\phi}$. For a relatively strong poloidal field the rise is slower and therefore the flux ropes reach a lower height in the same amount of time. In certain cases the rise can be completely halted. It turns out that anti-parallel twisted ropes reach higher and faster. When it comes to the flux ropes' topology, the geometrical shape of ropes also depends on the strength and sign of the poloidal field: the apex of anti-parallel twisted ropes are flatter and have steeper magnetic gradients in their axial field.

Valeryj N. Kryvodubskyj presented the sign change of helicity parameter in the SCZ and observed meridional migration of the surface magnetic patterns. In the frames of the macroscopic magnetohydrodynamics in the mixing length approach, he investigated the helicity parameter of turbulent convection in the solar convection zone (SCZ) 
with taking into account the radial inhomogeneity of turbulent pulsations. Allowance for the sharp radial gradient in turbulent velocity results in a change of sign of the helicity parameter from positive to negative (in the northern hemisphere) and, as a consequence, in the formation of the negative $\alpha$-effect layer near the bottom of the SCZ (which has thickness about $30000-40000 \mathrm{~km}$ ). The mean negative helicity parameter amplitude (averaged over the negative values region) amounts to $2 \mathrm{~m} \mathrm{~s}^{-1}$. When taking into consideration a negative sign of $\alpha$-effect in deep layers, then the physical conditions at midto low-latitudes domain (where a radial gradient of angular velocity, derived from recent helioseismological measurements, has positive sign) are favourable for $\alpha-\omega$ dynamo waves to propagate toward equator producing the sunspots migration as the solar cycle progresses. Since at high latitudes the radial gradient of angular velocity has opposite sign, then here the excited dynamo waves must move toward the poles.

The effects of the shape and width of the tachocline on the butterfly diagram of the solar cycle was investigated by Gianantonio Guerrero, and Elisabete M. de Gouveia Dal Pino. Flux-dominated kinematic solar dynamo models, which have demonstrated to be quite successful in reproducing most of the observed features of the solar magnetic cycle, generally produce an inappropriate latitudinal distribution of the toroidal magnetic fields, showing fields of large magnitude in polar regions where the radial shear has a maximum amplitude. Employing a kinematic solar dynamo model, they explore the contribution of both the radial and the latitudinal shear in the generation of the toroidal magnetic fields by varying the shape and the width of the solar tachocline. They find that a prolate tachocline (as suggested by recent observations) is unable to resolve the aforementioned problem. On the other hand, they find that the latitudinal component of the shear term of the dynamo equation is always dominant over the radial component for producing toroidal field amplification, and that this field is suppressed at high latitudes if the tachocline has a width $d \leqslant 0.03 \mathrm{R}_{\odot}$ or $d \geqslant 0.1 \mathrm{R}_{\odot}$. The best fit to the observed butterfly diagram is found for a tachocline with width smaller than $3 \%$ of the solar radius. The authors note, however, that this result is somewhat sensitive to the adopted magnetic diffusivity. In the present work, they have adopted a diffusion profile for which the tachocline is approximately half sub-adiabatic and half turbulent. If taking, for instance, a tachocline with a smaller turbulent portion, then its width could be a slightly larger than the value above.

\section{Photospheric magnetic field}

The fine structure of the sunspot penumbra is an ideal target to study the effects of radiative magnetoconvection in inclined magnetic field and to compare theoretical models with observed properties. Rolf Schlichenmaier presented the spectroscopic and spectro-polarimetric measurements, which are interpreted to reconstruct the thermal stratification and the complex topology of the magnetic field and the flow field. Such measurements reveal that the line-of-sight velocity and the magnetic field depend on depth in the atmosphere and exhibit gradients or discontinuities. The magnetic field is found to be uncombed, with an essentially horizontal component that carries a radial outward flow (Evershed flow), and a less inclined magnetic field component. He referred to the discovery of dark-cored bright filaments and presented spectroscopic measurements, which demonstrate that the dark cores are associated with the Evershed flow.

Debi P. Choudhary, and K.S. Balasubramaniam showed that the inclined magnetic fields beyond the penumbra appear as Moving Magnetic Features (MMF) that do not rise above upper photospheric heights. The dynamical properties of the moving 
magnetic features of the active region NOAA 9662 observed on 16 and 17 October 2001 using the Advanced Stokes Polarimeter (ASP) at Richard B. Dunn Solar Telescope of National Solar Observatory showed that spatial and temporal properties of the MMF filed is convoluted.

William C. Livingston, John W. Harvey, Olena Malanushenko, and Larry Webster showed that the sunspot magnetic fields can be as high as $6000 \mathrm{G}$. Their estimate is based on the fact that the photoelectric magnetographs underestimate field strength in sunspot umbrae because of scattered light. Two techniques that do work are photographic spectra or visual observations where the Zeeman $\sigma$-components are matched by the observer with the Hale-Nicholson tipping plate and the plate angle is converted to the field strength. They studied field measures of 32000 spots, years 1917 to 2004, from the archives of Mt. Wilson, Potsdam, Rome, and Crimea and found 58 spot groups with fields of $4000 \mathrm{G}$ or more. A careful look at the time history of equipment used at Mt. Wilson reveals that the non-linearity of the glass plate at large tilt angles was not taken properly into account, perhaps because of their rarity. When they correct the Mt. Wilson values for strong fields they find several cases of $5000 \mathrm{G}$ and one of $6100 \mathrm{G}$. Often such strong fields are associated with the presence of light bridges, but not always.

The simultaneous full-Stokes imaging polarimetry observations in Fe I 6303 and $\mathrm{H} \alpha$ was presented by Yoichiro Hanaoka. The Solar Flare Telescope of NAOJ, Mitaka, has been observing the photospheric magnetic field in the Fe I 6303 line with the imaging polarimetry technique for more than 10 years. Recently, they replaced the polarimeter for the Fe I 6303 observation with a high-sensitive one, using ferroelectric liquid crystals (FLC). Another FLC polarimeter was also installed into the $\mathrm{H} \alpha$ imager of the Solar Flare Telescope. Their FLC polarimeters have the polarization sensitivity of the order of $10^{-4}$, and with this sensitivity not only substantial improvement of the quality of photospheric vector magnetograms, but also detection of the full-Stokes polarization signals in the $\mathrm{H} \alpha$ line were realized. Now, they are operating simultaneous full-Stokes imaging polarimetry observations in the photosphere and in the chromosphere, and the three-dimensional information of the structure of the magnetic field can be obtained.

The other important aspect of determining the magnetic field from polarimetry observations are the inversion techniques. Julio Cesar Ramírez Vélez, Arturo López Ariste, and Meir Semel presented the spectropolarimetric observational data and the correspondent magnetic field inversion of the solar photosphere in quiet regions. The presence of atomic coupling between the nuclei momentum $I$ and the total electron momentum $J_{\mathrm{LS}}$, known as hyperfine structure, atomic regime where the total momentum $F=I+J$, was found in the observed data for the atom Mn I at $553.7 \mathrm{~nm}$. The signature of these hyperfine structure is clearly detected in circular polarisation in form of protuberance in the central part of the profile. They have developed an inversion code of the magnetic field using the Principal Component Analysis (PCA). A statistical test is applied to the code obtaining satisfactory inversion results. The inversion of the observed profiles, using the circular polarisation $V$ and the intensity $I$, shows mixed regions with values of $B$ higher than $800 \mathrm{G}$ and lower than $600 \mathrm{G}$ associated with the network and internetwork respectively.

Lofti Yelles Chaouche, Mark Cheung, Andreas Lagg, and Sami K. Solanki presented simulations of flux emergence in the solar photosphere diagnostics based on 3D radiation-MHD. They investigate flux tube emergence in the solar photosphere using a diagnostic procedure based on analyzing Stokes signals from different spectral lines calculated in 3D radiation-MHD simulations. The simulations include the effects of radiative transport and partial ionization and cover layers both above and below the solar surface. 
The simulations consider the emergence of a twisted magnetic flux tube through the solar surface. They consider different stages in the emergence process, starting from the early appearance of the flux tube at the solar surface, and following the emergence process until the emerged flux looks similar to a normal bipolar region. At every stage they compute line profiles by numerically solving the Unno-Rachkovsky equations at every horizontal grid point. Then, following observational practice, they apply Milne-Eddington-type inversions to the synthetic spectra in order to retrieve different atmospheric parameters. They include the influence of spatial smearing on the deduced atmospheric parameters to identify signatures of different stages of flux emergence in the solar photosphere.

The quiet Sun magnetic field is an important ingredient in the solar magnetism, which was reviewed by Sami K. Solanki. The amount of magnetic flux in the quiet Sun, its distribution on the solar surface, its origin and evolution are topics that have been strongly debated in recent years. In JD03 he presented a critical overview of some of the results obtained from high-sensitivity polarimetric data and from numerical 3D radiationMHD simulations. He posed several important questions that are given in the concluding remarks of this article.

Continuing the topic of formation of small-scale plasma structures in the photosphere, Yu. V. Kyzyurov considered the possibility of formation of such structures in the turbulent flows of photospheric gas and to analyse the dependence of their spectrum and intensity on height and on the strength of magnetic field. Results of observations with high-spatial resolution clearly show that the solar photospheric flows include both organized and stochastic motions. The spectra associated with the stochastic velocity fields obey power laws, which are close to the law of Kolmogorov turbulence. Since the degree of photospheric gas ionization is small, electrically charged particles can be regarded as passive contaminants embedded in motions of the gas. Taking these facts into account, the present consideration was based on the macroscopic description of the charged particle behaviour in the ambipolar diffusion approximation, and under the given velocity field of the neutral gas. Length-scales of the plasma structures were restricted to an inertial range of the turbulence, where the random velocity field of the gas is homogeneous and isotropic one with the known statistical properties. An analytic expression for the spatial spectrum of the structures was derived as well as the formula for estimation of the RMS level of their intensity. The plasma structure formation was analysed in the height range from 150 to $350 \mathrm{~km}$. It was shown that in spite of changes in parameters of the photosphere and the turbulence with altitude (e.g., an increase in the kinematic viscosity of the gas, and decrease in the rate of turbulent energy dissipation), the shape of the spectrum and the intensity of the structures remain almost unchanged in the calculations. At the same time, the increase in the magnetic field from 5 to $250 \mathrm{G}$ results in the increase in the intensity of deviation from the mean plasma density from 2.5 to $6 \%$ for the length-scales of structures smaller than $300 \mathrm{~km}$, and in the slope of the spectrum, if it is approximated by a power law, then power index takes values from -1.2 to -1.6. The obtained result seems to be important for better understanding of basic solar phenomena, such as generation of the random component of magnetic field or chaotic excitation of solar oscillations.

Transport of solar magnetic elements in the intergranular lanes was presented by A.A. Stanislavsky, and Dmytro V. Mukha. The Sun is an excellent natural laboratory for studying the nonlinear plasma phenomena. Photospheric convective motions of magnetic flux elements cause much of the solar surface phenomena. Using the observed solar data to estimate diffusion properties of photospheric bright points for times periods less than 20 minutes, they construct a transport model describing a sub-diffusive motion of solar 
magnetic elements in the intergranular lanes. The anomalous diffusion arises from the probabilistic distribution of waiting times attracted to a stable law. This suggests an analytical explanation of this observations and a revision of the Leightons model.

Observations of magnetic fields in the quiet Sun indicate that kilogauss-strength fields can be found in the intergranular lanes. Since the magnetic energy of these localised features greatly exceeds estimates of the kinetic energy of the surrounding granular convection, it is difficult to see how these features could be formed simply by convective flux concentration. Paul J. Bushby presented super-equipartition fields in simulations of photospheric magnetoconvection. Idealised, high-resolution simulations of three-dimensional compressible magnetoconvection are used to investigate the formation of these features numerically. Initially he takes a fully developed non-magnetic convective state into which he inserts a weak, uniform, vertical magnetic field. Magnetic flux is rapidly swept into the convective downflows, where it is concentrated into localised regions. As the field strength within these regions becomes dynamically significant, the high magnetic pressure leads to partial evacuation (via the convective downflows). This process continues until the field is strong enough to locally suppress convection. Provided that the magnetic Reynolds number is large enough, the strength of the resulting magnetic fields significantly exceeds the (so called) "equipartition" value, with the dynamical effects of the surrounding convection playing an important role in confining these magnetic features to localised regions. These results can be related to the well-known convective collapse instability, although there are some important differences between the two models. Although this is only an idealised representation of photospheric magnetoconvection, super-equipartition magnetic fields are a robust feature of these simulations. Therefore, the existence of kilogauss-strength magnetic fields in the quiet Sun is certainly qualitatively consistent with the findings of this model.

Katarzyna Mikurda, and Christian Beck presented the observational evidence for the "hot wall" effect in small magnetic flux concentrations. When lacking polarimetric observations, the bright points (BPs) visible in the G-band at $430 \mathrm{~nm}$ are commonly used as tracers for magnetic fields. Observations were taken on October 11, 2005 at the German Vacuum Tower Telescope and involved the Tenerife Infrared Polarimeter (TIP) at $1.5 \mu \mathrm{m}$, the Telecentric Etalon Solar Spectrometer (TESOS) in the Fe I spectral line at $557.6 \mathrm{~nm}$, and a speckle setup in G-band. The area scanned by TIP was $75^{\prime \prime} \times 33^{\prime \prime}$ and covered a pore surrounded by network. The TIP spectra were inverted with the SIR (Stokes Inversion based on Response functions) code to retrieve the magnetic field vector. They find that G-band BPs are not co-spatial with the central part of the flux concentrations. Even at the small heliocentric angle of 12 degree, BPs appear projected on the limb side walls of the granules, whereas the fields are concentrated in the intergranular lanes. Their findings indicate that the G-band bright points are a result of the hot wall effect. The downward shift of the optical depth scale in the presence of magnetic fields allows to see deeper and hotter layers, where the $\mathrm{CH}$ molecule dissociates, in the granules next to the field concentrations. Thus, information drawn from the observations of BPs cannot be used to conclude on the actual variation of the magnetic field structure, as only the outer parts of the flux concentrations are seen in the BPs.

\subsection{Photospheric magnetic field and flares}

The photospheric magnetic field has been extensively used to study the solar flares. The magnetic field configuration and evolution of a highly flare-productive region NOAA 10808 was presented by Takako T. Ishii, Kaori Nagashima, Hiroki Kurokawa, Reizaburo Kitai, Satoru Ueno, Shoichi Nagata, and Kazunari Shibata. Active 
regions on the Sun have different flare productivities. Some regions produce many large flares, while others produce no flares. The key factor of a high flare productivity is the complexity of magnetic field configuration of the region. They found that the twisted structure of emerging magnetic flux bundles is the essential feature of flare-productive active regions. The active region NOAA 10808 showed the highest flare activity during the current solar cycle (cycle 23) in September 2005. They studied the formation process of delta-type magnetic configuration using SOHO/MDI magnetograms and flares using TRACE data. They also studied the evolution of magnetic shear and $\mathrm{H} \alpha$ filaments using $\mathrm{H} \alpha$ full disk images and full disk vector magnetograms obtained with SMART. They summarize the characteristics of magnetic field configuration of this region and discuss the relation between the configuration and the high flare activity.

The flare-related magnetic field evolution was studied by S.N. Chornogo, and N.N. Kondrashova, who modelled the photospheric layers to study their physical state in two sites of an active region about 30 minutes before and in the onset phase of the solar tworibbon 2N/M2 flare. The soft X-ray enhancement and microwave bursts occurred prior to and during the flare. The models derived from the inversion reproduce the spectral observations in several Fraunhofer lines. All the lines are weakened to a various degree before the flare relative to those in the quiet photosphere spectrum. In the initial phase of the flare they became still more weak and wide. The models consist of two atmospheres: the magnetic flux tube and the ambient medium. The inferred models show the changes in all photospheric parameters both before and at the onset of the flare. A comparative analysis reveals the difference between photospheric physical conditions in two kernels. Before the flare the inner kernel located near the filament was cooler than the outer one. The flux-tube models show strong downflows in the outer kernel and upflows in the inner kernel in the deep photospheric layers. Upward motions of the surrounding photospheric material were found. In the impulsive phase of the flare the variations of the photospheric parameters manifested itself in the inner kernel more than in the outer one.

Magnetic fields and thermodynamic conditions at photospheric level of two solar flares, on July 19, 2000 and August 4, 2005 of importance M6.4/3N and C8.4/1N, respectively, were studied by V.G. Lozitsky, E.V. Kurochka, and O.B. Osyka using the echelle Zeeman spectrograms obtained with horizontal solar telescope at the Astronomical Observatory of the Kyiv Shevchenko National University. The Stokes $I \pm V$ profiles were compared for about ten lines of Fe I, Fe II, Cr II, Sr II, and Ti II. The direct magnetic field measurements using the "center of gravity" method has shown the non-monotonous magnetic field gradient in the area of the flare, in contrary to usual weak negative gradient outside flare emission. Semi-empirical models of flares, calculated close to the maxima using Baranovsky's program (Baranovsky 1993), are in good agreement with these direct measurements. In particular, for the flare of July, 2000, a very narrow $(\leqslant 100 \mathrm{~km})$ and sharp magnetic field peak at the photospheric level $\left(\log \tau_{500} \approx-2\right)$ was discovered. This peculiarity indicates likely the local magnetic field amplification due to some specific process during the flare. Another interesting detail are two discrete hot flare layers related to the middle photosphere and temperature minimum zone. The turbulent velocities had one or two peaks placed close to the mentioned peaks of temperature. In the weaker flare on August 4, 2005 the magnetic field increased with the depth in the photosphere without peculiarities.

Graham Barnes, Dana W. Longcope, Colin Beveridge, B. Ravindra, and Kimberly D. Leka employed the Minimum Current Corona (MCC) model to estimate the amount of magnetic free energy and helicity injected into the coronal magnetic field of an active region. In the MCC model, each concentration of photospheric magnetic flux 
is represented by a point source, greatly simplifying the magnetic topology. Advecting an initial partitioning of the flux through a long time series of magnetograms results in a persistent set of sources. They show that the centroid velocity of a partition compares well with the flux-weighted average over the partition of the local correlation tracking velocities. Flux domains, bundles of field lines interconnecting pairs of sources, are surrounded by separatrix surfaces. The intersection of two separatrices is a separator field line, which is the site of reconnection in this model. The evolution of the photospheric field causes the sources to also evolve, which would lead to changes in the domain fluxes to maintain a potential field configuration if reconnection could proceed rapidly. However, in the absence of reconnection, currents begin to flow to maintain the initial distribution of domain fluxes. The minimum energy state occurs when currents flow along the separators. The magnitude of the separator currents can be estimated and combined with geometrical properties of the separators to give a lower bound to the magnetic free energy of the system. The motion of sources about one another adds braiding helicity to the system, while the internal rotation of a partition adds spin helicity. Starting from an initial potential field configuration, changes in the free energy are presented for a time series of data for NOAA AR 8210 on May 1, 1998.

Build-up of a CME and its interaction with large-scale magnetic structures was presented by Lidia van Driel-Gesztelyi, Christopher P. Goff, Pascal Démoulin, J. Len Culhane, Karl-Ludwig Klein, Cristina H. Mandrini, Sarah A. Matthews, Louise K. Harra, and Hiroki Kurokawa. A series of flares (GOES class M, M and C) and a CME were observed on January 20, 2004, occurring in close succession in NOAA 10540. Types II, III and a N radio bursts were associated. They investigate the link between the flares (two impulsive flares followed by an LDE) and the CME as well as the origin of the rare decametric N-burst by using the combined observations from TRACE, SOHO-EIT, H $\alpha$ images from Kwasan Observatory, SOHO-MDI magnetograms, GOES and radio observations from Culgoora and Wind-WAVES as well as magnetic modelling to understand the complex development of this event. They find that there is a link between the first two impulsive flares to tether-cutting reconnections and the launch of the CME, while the last of the flares, an LDE, in the relaxation phase forced reconnections between the erupting flux rope and neighbouring magnetic field lines. They show that reconnection with the magnetic structure of a previous CME, launched about 8 hours earlier, injects electrons into open field lines having a local dip and apex of about 6 solar radii height. The dipped shape of these field lines was due to large-scale magnetic reconnection between expanding magnetic loops and open field lines of a neighbouring streamer. This particular situation explains the observed decametric N burst. This complex observation shows that impulsive quadrupolar flares can be eruptive, while an LDE may remain a confined event. They find that the reconnection forced by the expanding CME structure is followed by a relaxation phase, when reconnection reverses and restores some of the pre-eruption magnetic connectivities. The observed decametric N-burst was caused by the interaction of two CMEs and reconnection of their expanding magnetic field with neighbouring streamer field lines - a very particular interplay, which explains why N-bursts are so rare.

Yu Liu presented a study of halo coronal mass ejections and configuration of the ambient magnetic fields. He examined 104 halo CMEs in the time interval 2000-2004 with respect to the configuration of their ambient magnetic fields. He defines three types of halo CMEs in terms of characteristics of the ambient magnetic field where the CMEs occur: the CMEs occur under the heliospheric current sheet (type 1), in the open flux areas (type 2), and under the plasma sheet (type 3) and find that type 2 and 3 CMEs 
appear to be faster than type 1, which implies that configuration of the ambient magnetic field play a role in determining halo CMEs' speed. He also find a weak correlation between speed of type 3 halo CMEs and the peak X-ray flux of the associated flares, but no such correlations are found for types 1 and 2 . This suggests that kinetic characteristics of type $3 \mathrm{CMEs}$ are more directly related with properties of the associated flares. Considering the configuration of the ambient magnetic field of type 3 CMEs that possess much less closed flux for confining propagation of CMEs, it is suggested that the overall closed field works as an additional factor to shape propagation of a CME, while the associated flare might define the speed of this CME in the first place, as demonstrated in several current studies. It is not clear so far what roles the ambient field may play in initiating a CME. He also presented configuration of magnetic field related to fast halo CMEs. He found that the fast halo CMEs in October-November 2003 initiated from areas with special configuration of magnetic field such as open flux or plasma sheet. He concludes that the configuration of the background magnetic field plays an additional role in shaping the propagation of the fast halo CMEs, and thus ensure a high speed when special configurations apply. In his work he extends the study to a large sample: including all halo CMEs detected by SOHO-LASCO and identified by Gopalswamy group, to explore more general relationship between halo CMEs' propagation and configuration of background magnetic field.

Ayumi Asai, Takako T. Ishii, Kazunari Shibata, and Nat Gopalswamy showed the anemone structure of active region NOAA 10798 and related geo-effective flares/CMEs. They reported the evolution and the coronal features of an active region NOAA 10798, and the related magnetic storms by examining in detail the photospheric and coronal features of the active region by using observational data in soft X-rays, in extreme ultraviolet images, and in magnetogram obtained with GOES, SOHO satellites. They also examined the interplanetary disturbances from the ACE data. This active region was located in the middle of a small coronal hole, and generated 3 M-class flares. The flares are associated with high speed CMEs up to $2000 \mathrm{~km} \mathrm{~s}^{-1}$. The interplanetary disturbances also show a structure with southward strong magnetic field. These produced a magnetic storm on August 24, 2005. They conclude that the anemone structure may play a role for producing the high-speed and geo-effective CMEs even at the near-limb locations.

Hong-Qi Zhang presented an observational study of solar magnetic field and eruption phenomena and the development of new optical instrumentation at Huairou Solar Observing Station, National Astronomical Observatories of China. They find that the synthetical analysis between photospheric vector magnetic field and the morphological configuration in solar atmosphere provides the essential information on the developments of magnetic energy in source regions of flare-CME eruptions. For example the observational study on the evolution of photospheric vector magnetic field provides the basic information for the emergence, storage, relaxation of non-potential magnetic energy in solar active regions (such as, NOAA 9077, 10488 and 10720), and the relationship with the trigger of solar flare-CMEs. The photospheric vector magnetic field, inferred from the observations, also provides the possible formation mechanism of helical magnetic configuration and transferring form in the solar atmosphere into the interplanetary space.

\section{Chromospheric magnetic field}

As the flux rises into the chromosphere it expands to maintain the pressure balance in a decreasing gas pressure environment. The expanded chromospheric magnetic field creates a canopy. John W. Harvey reviewed the properties of chromospheric magnetic field by 


\section{Extended canopies around active regions}

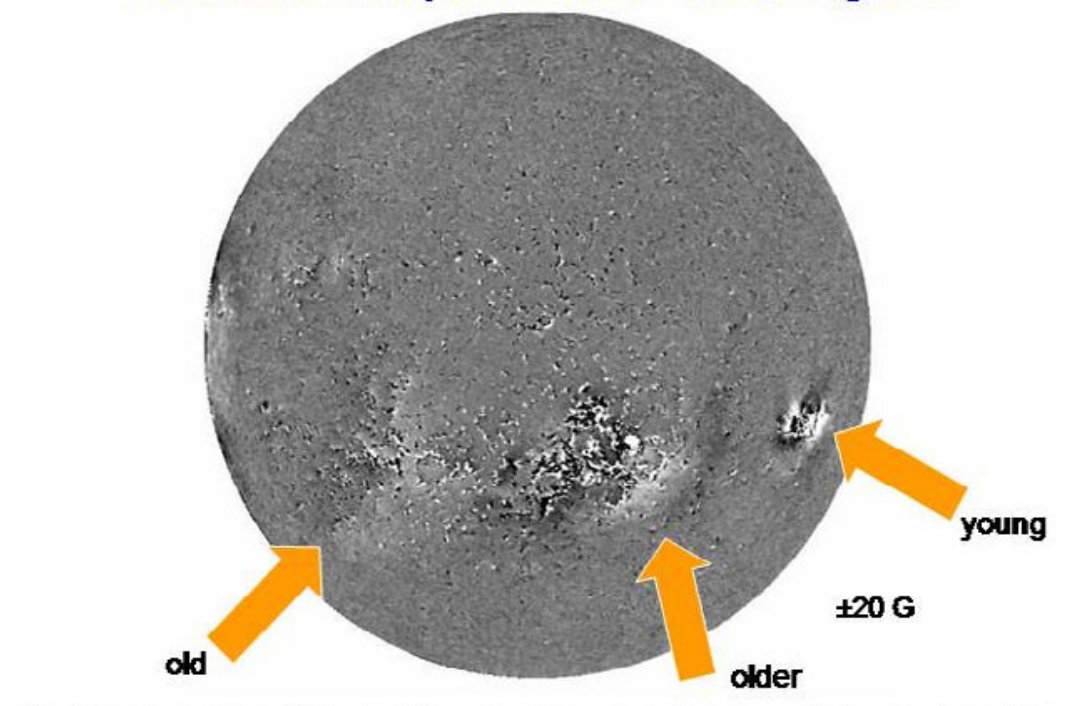

Around all active regions, young and old; mainly horizontal

NSOrSOUS 20040008

Figure 3. The extended canopy in chromospheric magnetogram.

using a decade of regular full-disk observations of the longitudinal Zeeman splitting of the chromospheric $854.2 \mathrm{~nm}$ Ca II line that reveals many interesting magnetic phenomena. The most prominent is a large-scale, mainly horizontal diffuse field surrounding active regions. This extended canopy field is not present in the photosphere and is closely related to chromospheric fibrils and the circumfacule phenomenon. Chromospheric canopy fields are also found around small magnetic concentrations. The larger patterns endure as long as active regions can be recognized as distinct entities. The typical field strength is a few Gauss. The direction is mainly horizontal with azimuth usually, but not always, directed away from the strongest nearby fields. The atmosphere in chromospheric canopy regions has different radiative, dynamic and structural properties than other regions of the chromosphere. Also prominent are magnetic channels which are closely related to filament channels. Their properties are similar to the chromospheric canopies but the azimuth of the field frequently has a strong component along the axis of the channel. Filaments are frequently but not always seen in magnetic channels. On the largest scale, there are weak areas $(\sim 1 \mathrm{G})$ of radially oriented fields that are observed when many active regions are simultaneously present. The $854.2 \mathrm{~nm}$ line shows strong spatial variations including core emission. Calculating the longitudinal field with the traditional center-of-gravity scheme gives incorrect results. The field is estimated by comparing the observed local $\mathrm{V}$ profile with the wavelength derivative of the local I profile at each pixel. As shown in Fig. 3 the chromospheric magnetogram shows extended canopy where the magnetic field is mostly horizontal irrespective of whether the active region is old or young. How does the quiet region flux rise in solar atmosphere? Do quiet Sun flux tubes poke upward through the horizontal canopy fields that extend far away from active regions (more 
inter-mingling of different field components)? The future vector magnetic field observations with high spatial resolution might hold the answer.

The chromospheric small-scale solar magnetic features were studied with high-resolution CN spectroscopy by Vladimir V. Zakharov, Achim Gandorfer, and Sami K. Solanki. The high-resolution spectroscopic observations of the Sun have been carried out with the TRIPPEL spectrograph installed at the new 1-m Swedish Solar Telescope (SST) using real-time adaptive optics correction. A detailed spectroscopic analysis of individual photospheric bright points (BP) and faculae-like structures simultaneously in two spectral domains, i.e., $387.588<\lambda<388.473 \mathrm{~nm}$ (violet $\mathrm{CN}$ band) and in a blue spectral band at $436.1<\lambda<436.9 \mathrm{~nm}$, containing absorption lines of $\mathrm{CH}$, obtained at the disc center and near the limb is presented. The estimated spatial resolution of the obtained spectra is around $0.25^{\prime \prime}$ while the spectral resolving power is around 130.000 in the first domain, and 76.000 in the second spectral region, respectively. The first spectral band covers absorption lines of both, $\mathrm{CH}$ and $\mathrm{CN}$ molecules, as well as many atomic lines. This made it possible to perform a quantitative comparison of their absorption and Doppler shifts in the different photospheric features. The absorption lines of the CN molecule and many atoms are depressed in a BPs interior with respect to those in the quiet Sun. A quantitative comparison of the relative line depression of $\mathrm{CH}$ lines with respect to $\mathrm{CN}$ lines showed that the latter have weaker absorption by a factor of 1.28 at the disc centre and 1.32 near the limb. The CN line-core intensity, at the disc centre, has higher BP contrast than the contrast in the $\mathrm{CH}$ line-core by a factor of 1.9 , and the ratio of these contrasts is decreasing with increasing continuum intensity of the BPs. This trend is similar to that obtained from previous simultaneous G-band and violet CN-band imaging observations. Measurements of contrasts and rms contrasts of line-core, integrated and local continuum intensities are provided. Analysis of Doppler shifts and line broadening of an Fe I line at $387.777 \mathrm{~nm}$ revealed an increase of the FWHM in the BPs interior and in dark intergranular lanes and a decrease with increasing intensity of the granules. The first results of a direct comparison of observed CN spectra with those simulated in MHD models in different photospheric features are presented.

The challenges for chromospheric spectropolarimetry were reviewed by K.S. Balasubramaniam. The solar chromosphere is the most immediate and intermediary layer where competitive magnetic forces begin to dominate their thermodynamic counterparts. The chromosphere quickly responds to magnetic energy release processes, and is one of the early indicators of this energy release. Hence, understanding the nature of the magnetic field at the chromospheric layer is vital. Measurements of the chromospheric magnetic field using spectropolarimetry are necessary to constrain 3D models of the magnetic field geometry via extrapolation methods. In the review he described the challenges for measuring and inferring the chromospheric magnetic field in solar-disk measurements.

One of the important chromospheric lines used for the magnetic diagnostic is He I 1083.0 nm. Yulia S. Zagaynova, Pavel G. Papushev, and Sergey A. Chuprakov investigated the He I 1083.0 triplet profile in sunspot umbrae. This triplet gives an unique view of the chromosphere in active regions. Several articles at early time had indicated the enhancement of the absorption at this line in sunspot umbra among other features. The line depths of the He I $1083.0 \mathrm{~nm}$ triplet are compared with plages and other AR features reliably registered in sunspot umbrae. It is a good opportunity for examination of the chromospheric heating processes connected with quite different magnetic structures. Up to date, systematic investigation of helium spectrum in sunspot umbrae has not been conducted. Statistical results of the He I $1083.0 \mathrm{~nm}$ triplet line observations in sunspot 
umbrae are presented. The observations were made at the large Nicolsky coronagraph at Sayan mountain observatory. The setup for the multi-spectral observation was used. This setup consists of the CCD spectrograph and the two bandwidth $(\mathrm{He} / \mathrm{H} \alpha)$ birefringent filter. The lens system of the coronagraph provides the possibility for synchronous registration of the spectral line and two monochromatic images in quite different spectral regions. A method to avoid blurring effects on line profiles based on the point spread function (PSF) has been developed. PSFs have been derived from a pore continuum contrast profile, registered at the same time and place, and from a set of empirical data. These PSFs were used to restore monochromatic images. More than 35 umbral spectra of sunspots with areas from 1 to $10^{6}$ millionths have been recorded. The currently available data is not rich enough but some implications are available. It can be stated that He I 1083.0 triplet really exists in sunspot umbrae and its line depth, equivalent line width, and half-width are increasing with sunspot area. For leading and individual unipolar sunspots, the triplet dependences between line parameters and spot areas have been found which are quite different from those obtained for following spots.

Inferring the magnetic field from He I $1083.0 \mathrm{~nm}$ is complex. Clementina Sasso, Andreas Lagg, and Sami K. Solanki successfully used the multi-component analysis of a flaring region in the chromospheric He I $1083.0 \mathrm{~nm}$ triplet. They present infrared spectropolarimetric observations of the solar active region NOAA 10763 obtained with the new Tenerife Infrared Polarimeter (TIP 2) at the German Vacuum Tower Telescope (VTT) during May 2005. The region was in the initial phase of a C2.0 flare. They observe up to 4 atmospheric components within the spatial resolution element. The components are clearly separated in wavelength, the largest separation corresponding to downflow velocities of up to $64 \mathrm{~km} \mathrm{~s}^{-1}$. They give preliminary results on the magnetic vector and the line-of sight velocity obtained by inverting the Stokes $I, Q, U$, and $V$ profiles of the He I $1083.0 \mathrm{~nm}$ triplet.

Even quiescent solar prominences may become active and sometimes erupt. These events are occasionally linked to coronal mass ejections. However we know very little about the plasma properties during the activation and eruption processes. The spectral line of helium in chromosphere and transition heights is an important part of magnetic diagnostic of these phenomena. The first computations of the helium line profiles emitted by an eruptive prominence were presented by Nicolas Labrosse, Jean-Claude Vial, and Pierre Gouttebroze. The prominence is modelled as a plane-parallel slab standing vertically above the solar surface and moving upward as a solid body. The helium spectrum is computed with a non local thermodynamic equilibrium radiative transfer code. The effect of Doppler dimming or brightening is investigated in the resonance lines of HeI and HeII formed in the EUV, as well as in the HeI $1083.0 \mathrm{~nm}$ and HeI $587.6 \mathrm{~nm}$ lines. They focus on the line profile properties and the resulting integrated intensities. They also study the effect of frequency redistribution in the formation mechanisms of the resonance lines. It is shown that the helium lines are very sensitive to Doppler dimming effects. Together with the hydrogen lines they offer the possibility of a powerful diagnostic of the active and eruptive prominence plasma.

Pavel Papushev, and Rafik Salakhutdinov presented the evolution of chromospheric spicules. This well-known phenomenon observed in the solar chromosphere has been subject of a long-standing investigation both observationally and theoretically. At last few years, another theoretical approach has been proposed. It is the leakage of p-mode into the chromosphere. This approach is based on the observation of spicules on the solar disk in the enhanced network. They demonstrated wave-like fluctuations with duration much less than spicules' lifetime (with a period less than $100 \mathrm{~s}$ ), anomalous half-width of 
spectral lines, and limb spicules' transversal motion variations, which time spectrum is very similar to that of a line-of-sight velocity. These phenomena need to be explained.

Merging of filaments in a dual-filament system was presented by Katarzyna Mikurda, and Sara F. Martin. The dual filament system merges to form one extended filament. The filaments were observed at Helio Research in multiple wavelengths around $\mathrm{H} \alpha$ using a tunable filter and a narrow band Fabry-Perot etalon as part of the Joint Observing Campaign. These observations are used to create two-dimensional Dopplergrams. The $\mathrm{H} \alpha$ images are compared with data taken onboard the SOHO (Solar and Heliospheric Observatory) spacecraft (EIT at $304 \AA$ and LASCO C2). The GONG magnetograms provide the information on photospheric magnetic fields. The filaments were observed on the solar disk between October 10 and 16, 2004. The morphology of the filament system in $\mathrm{H} \alpha$ and He II line at $304 \AA$ was compared and it was found that there is no clear evidence of an eruption associated with the merging of the filaments from either EIT or LASCO C2 in contrary to some previous findings.

As the magnetic structure explodes, the energy is released in chromosphere. Jing $\mathbf{P}$. Li, and Ming-De Ding showed the detection of explosive chromospheric evaporation during a two-ribbon flare. They presented two-dimensional spectral observations of an $\mathrm{X} 1.2$ two-ribbon flare in which blue asymmetry of $\mathrm{H} \alpha$ profiles are found at one kernel for more than 1 minute. It is interesting that this kernel seems to move along a flare loop seen on the Transition Region and Coronal Explorer (TRACE) images since its appearance. While on the loop footpoints, the $\mathrm{H} \alpha$ profiles are found to be red shifted. The statistical distribution and the spatial distribution of the blue asymmetry at the kernel are also studied. There seems to be a counterpart of the kernel on TRACE $1600 \AA$ images. They calculate the moving speed of the kernel by measuring its moving front on TRACE images and find that it has a good temporal correlation with the line-ofsight velocity calculated from the $\mathrm{H} \alpha$ profiles using a wing bisect method. The maximum speed of the kernel measured from TRACE images is $\sim 200 \mathrm{~km} \mathrm{~s}^{-1}$, which is similar to the chromospheric evaporation speed deduced from soft X-ray lines. Suppose that this kernel may be the plasma evaporated from the chromosphere, it will be the first time to get a direct evidence that supports the scenario of chromospheric evaporation and condensation of solar flares in $\mathrm{H} \alpha$ line.

A study relating the solar differential rotation and helicity of magnetic clouds was presented by Katya Georgieva. Magnetic clouds - a subclass of coronal mass ejections distinguished by the smooth rotation of the magnetic field inside the structure - are the most geo-effective solar drivers. The rotation of the magnetic field in magnetic clouds reflects the rotation in their source region on the Sun whose quantitative measure is the magnetic helicity. The helicity in the corona is determined by two factors: the helicity transferred from the solar interior and the helicity generated by the surface differential rotation. The helicity transferred from the interior into the corona is proportional to the squared magnetic flux (maximum in sunspot minimum and minimum at sunspot maximum), and is equal in magnitude and opposite in sign in the two solar hemispheres: positive in the south and negative in the north, independent of the magnetic polarity cycle. The surface differential rotation, fastest at the solar equator and decreasing toward the poles, generates helicity with the same sign: negative in the north and positive in the south. Therefore, magnetic clouds originating from the northern solar hemisphere should contain negative helicity, while the ones originating from the southern hemisphere should have positive helicity, with the total negative helicity over the solar cycle equal to the total positive helicity. However, this "hemispheric helicity rule" is true in only $70-80 \%$ of the cases. Moreover, in the last solar cycle the net helicity carried away by magnetic 
clouds was found to be negative, and the number of the left-handed magnetic clouds was higher than of the right-handed ones. She studies the solar differential rotation and finds that in cycle 23 the rotation rate was higher and the rotation was more differential in the northern solar hemisphere, consistent with the excess negative helicity carried away by magnetic clouds. She investigates 39 magnetic clouds associated with major geomagnetic storms in the last solar cycle (1997-2001) whose solar sources have been identified. In 27 of them (73\%) the magnetic cloud observed at the Earths orbit with the expected helicity. In 10 out of the 12 cases of violation of the hemispheric helicity rule, the differential rotation in the source region of the magnetic cloud is found to be of the "anti-solar type".

Masaoki Hagino, Reizaburo Kitai, and Kazunari Shibata studied the helicity sign of 239 intermediate filaments (IFs) on the solar disk from 2005 July 1 to 2006 May 15. The intermediate filament usually locates between two active regions. $\mathrm{H} \alpha$ images used were obtained with the Solar Magnetic Activity Research Telescope (SMART), located at the Hida Observatory of Kyoto University. The distribution of the chromospheric helicity shows a hemispheric tendency; namely, the filaments in the northern (southern) hemisphere tend to show a dextral (sinistral) barbs, respectively, in agreement with previous studies. On the other hand, the EUV filament helicity was determined from the SOHO-EIT data. Using both data, normalized helicity of intermediate filaments was derived. The latitude distribution of normalized helicity shows negative slope. Moreover, 12 filament eruptions were found in the data. The 7 of 12 filaments show the clear opposite sign to the hemispheric tendency of the magnetic helicity. Figs. $4 a$ and $b$ show a latitude distribution of dextral and sinistral IFs in H $\alpha$. The helicity of 239 IFs (110 in the north hemisphere, 129 in the southern hemisphere) was studied and it was found that $71 \%$ (78 of 110) of IFs in the northern hemisphere have dextral barbs and 67\% (87 of 129) of filaments in the southern hemisphere have sinistral, which agreed with the well-known hemispheric tendency of the filament barbs. Figs. $4 c$ and $d$ show a latitude distribution of positive and negative chirality in EUV IFs. The helicity pattern of EUV filaments obtained with SOHO-EIT $171 \AA$ also shows a similar hemispheric tendency. Namely, $65 \%$ (71 of 110) of EUV filaments in the northern hemisphere exhibit negative helicity and the $65 \%$ (84 of 129) of filaments in the southern hemisphere show positive helicity. Using both data above, normalized helicity of IFs was derived. The normalized helicity depends on the ratio $L / \varpi$ and the pitch angle $\gamma \cdot\langle L / \varpi\rangle \approx 30$ in the IFs data. Due to the long sinuous structure, these IFs aspect ratio may be smaller (Rust \& Kumar (1994)). $\langle|\gamma|\rangle \approx 35^{\circ}$. The normalized helicity was derived for 181 IFs where it was possible to determine the helicity sign. The slope of linear fit is $d H / d \theta=-0.11 \pm 0.06 \mathrm{deg}^{-1}$, which is negative, in agreement withprevious studies measured in active regions (Hagino \& Sakurai 2004, etc.).

\section{Coronal magnetic field and eruptive phenomena}

For a given photospheric magnetic field distribution, the field topology can be derived using the models. The magnetic field is thought to be the source of the energy released in diverse observed coronal phenomena, from the less energetic coronal heating to the most violent flares and prominence eruptions. These phenomena involve not only very different scales from the energetic, but also from the temporal point of view. Magnetic field reconnection, which is efficient only at very small spatial scales, has been the energy release mechanism so far proposed. Cristina H. Mandrini reviewed the progress in understanding the magnetic field at higher solar atmospheric layers with photospheric 

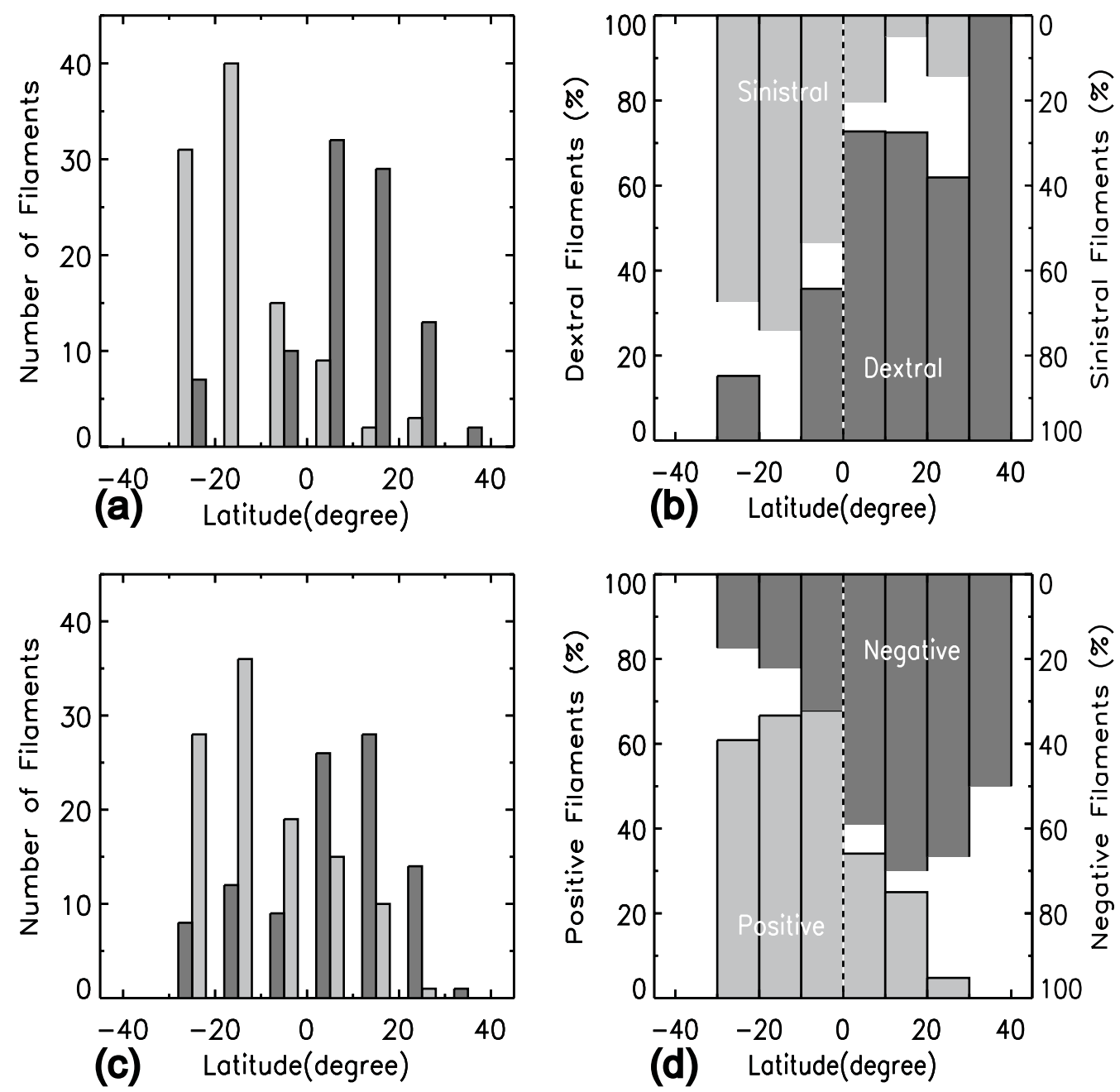

Figure 4. Latitude distribution of helicity sign of intermediate filaments. (a) Number of dextral (dark-shade bar) and sinistral (light-shade bar) $\mathrm{H} \alpha$ filaments observed by SMART H $\alpha$ data. (b) Relative frequency of helicity for every latitudinal zone. (c) Number of negative (dark-shade bar) and positive (light-shade bar) EUV filaments observed by SOHO-EIT. (d) Same as in (b), but from EUV filaments.

boundary condition. From a theoretical point of view, magnetic configurations with a complex topology, i.e., having separatrices, are the ones where current sheets can form in $2 \mathrm{D}$. When going to 3D, and if the photospheric magnetic field is described by a series of isolated polarities (surrounded by field free regions), a complete topological description is given by the skeleton formed by null points, spines, fans and separators, and associated separatrices (see the review by Longcope 2006 and the examples therein discussed). However, if the photosphere is fully magnetized, most of the above topological structures disappear: only separatrices associated to coronal magnetic nulls remain. Separatrices of a different origin are linked to the field lines curved up at the photosphere, defining the bald-patch locations (Titov et al. 1993). For some observed magnetic configurations, those topological structures are enough to understand where flare brightenings appear as a result of magnetic field reconnection. Few examples have been found where coronal null points, computed using either subphotospheric sources or magnetic field extrapolations 
to represent the coronal field, were associated with coronal activity (see e.g. Mandrini et al. 1991; Gaizauskas et al. 1998; Aulanier et al. 2000; Fletcher et al. 2001a; Mandrini et al. 2006). Concerning bald patches, they have been only found in connection with low energy release events: small flares (Aulanier et al. 1998), EUV brightenings (Fletcher et al. 2001b), and chromospheric events (Mandrini et al. 2002; Pariat et al. 2004).

The analysis of the topological structure of numerous active regions has shown that flares occur in a larger variety of configurations than those just discussed (see, e.g., Démoulin et al. 1994). Quasi-separatrix layers (QSLs) (Priest \& Démoulin 1995; Démoulin et al. 1996; Titov et al. 2002), which are regions where there is a drastic change in field-line linkage, generalize the concept of separatrices to magnetic configurations without magnetic null points and/or bald patches. Using coronal magnetic field models, QSLs have been computed in the largest variety of observed magnetic configurations (Démoulin et al. 1997; Mandrini et al. 1997; Fletcher et al. 2001b; Bagalá et al. 2000, and references therein). QSLs have been found located in coincidence with chromospheric and coronal loop brightenings of varied intensity, these brightenings could be also connected by field lines in the way expected by magnetic reconnection theory. Moreover, in cases where vector magnetograms were available, photospheric current concentrations were also located at the photospheric trace of QSLs. Recently, to relate QSLs with the formation of strong current concentrations and study the characteristic of the reconnection process occurring at QSLs, 3D MHD simulations of characteristic observed field distributions and photospheric motions have been developed by Aulanier et al. (2005) and Aulanier et al. (2006). The results from these simulations imply that electric currents at QSLs are amplified in time only if the QSLs are broader than the dissipative scale length, what was suggested in the previous observational analysis. Magnetic reconnection at QSLs occurs when the self-pinching of the current layers is strong enough to efficiently enhance the dissipation term in the induction equation. A property of this reconnection process is the continuous slippage of field lines along each other as they pass through the current layers. Their reviewed examples of observed regions showing varied levels of activity and their topologies, teach us that magnetic reconnection can occur in magnetic configurations with much wider topological characteristics than traditionally thought.

The 3D topology structure of coronal magnetic field was studied by Hui Zhao, Jing Xiu Wang, Jun Zhang, and Chi Jie Xiao. They used the Poincaré index of isolated null-points in vector field to strictly identify the 3D null-points in coronal magnetic fields reconstructed from observed vector magnetograms of several important active regions. Based on the null-points they identified, they revealed the essential topology skeletons of $3 \mathrm{D}$ coronal magnetic fields. Comparing these skeletons with images of $\mathrm{H} \alpha$, SOHO-EIT, TRACE, and X-ray, they found that the 3D topology structures of coronal magnetic fields are closely associated with solar flares and CMEs. They also found an indication of 3D magnetic reconnection by studying temporal series of the 3D topology structures. The thorough investigation of the 3D topology structures of coronal magnetic fields is a key to understand the physical mechanism of solar activity.

The effect of strong MHD discontinuities on the solar magnetic field was discussed by S.A. Grib who studied different cases of the collisions between the solar otational MHD discontinuities, going through the flux tubes, and the contact discontinuity, describing the solar transition region. It is shown in the frame of non-linear MHD problem of Riemann that both a fast and a slow shock waves may appear in the solar corona. By the way the first falling rotational discontinuity being 3-dimensional helps the 2-dimensional (described by the theorem of complanarity) shock wave to appear as a refracted one. Thus the behaviour of the magnetic field is changed in the result of the splitting of 
an arbitrary discontinuity. The catastrophic case of the interactions of the solar MHD discontinuities due to the nonlinear character is indicated.

\section{L.T. Song, Cheng Fang, Yu-Hua Tang, Shi Tsan Wu, and Yang A. Zhang} described a fast extrapolation method for reconstructing the coronal nonlinear force-free field which is an improved method of upward integration to reconstruct the nonlinear force-free field (NFFF) in the solar corona. The method of upward integration can be modified to a well-posed one in the following way: Instead of using finite difference to express partial derivatives, smooth continuous functions are used to approximate the magnetic field. Three field components are expressed by amplitude functions multiplying morphology functions, and the four basic NFFF equations can be reduced to ordinary differential ones. They are then solved in an asymptotic manner. Considering the physical meaning of the force-free parameter $\alpha$, they found a self-consistent compatibility condition for the boundary values. Furthermore, they propose a computation algorithm, similar to the usual time-dependent 2D MHD simulation scheme. This algorithm is steady and not sensitive to the noise in the magnetic field (in particular the transverse field) measurement, and is able to deal with concentrated photospheric currents. The code runs very fast in an usual PC-computer and lasts only 6 minutes for the mesh of $80 \times 60(\mathrm{x}: \mathrm{y})$ up to a height of $80(216000 \mathrm{~km})$. This new method provides a powerful tool to analyze the magnetic field property of solar active regions, and is useful for the prediction of solar activity.

The quasi-static sequences of 2D magnetic potential fields in barotropic ideal MHD flows was discussed by Dieter H. Nickeler, Miroslav Bárta, and Marian Karlický taking into account the equation of motion. The equation of motion is often neglected as the plasma $\beta$ is assumed to be very small in the regions above the photosphere. In contrast to that they analyse how magnetic potential fields do evolve in the frame of barotropic ideal MHD flows. Here neither the pressure gradient nor the equation of motion can be neglected. They show special solutions by solving the set of ideal MHD equations in the case of a quasi-static approach. This implies that the non-linear term in the equation of motion is neglected.

Jun Zhang, and Guiping Zhou reported the magnetic field evolution in a coronal hole using Big Bear Solar Observatory (BBSO) and SOHO observations exploring the magnetic evolution and temperature variation in a coronal hole during its appearing and disappearing phases. The results are: $(a)$ In the early phase of the coronal hole formation, more than $65 \%$ of the magnetic flux is negative, and the coronal temperature, estimated from the ratio of $S O H O$-EIT 195 and $171 \AA$ images, is $1.05 \times 10^{6} \mathrm{~K}$ in the hole; $(b)$ In the end phase of the hole, about $55 \%$ of the magnetic flux is negative, and the temperature increases to $1.10 \times 10^{6} \mathrm{~K}$. The results display that in a coronal hole there is a connection between magnetic evolution and coronal temperature and that the coronal temperature increases while the magnetic imbalance of the both polarities decreases.

\subsection{Coronal heating}

Coronal heating was another point of intense discussion. The adequacy of different models of the corona heating is discussed by Olga G. Badalyan, and Vladimir N. Obridko. The correlation between the brightness of the coronal green line Fe XIV $530.5 \mathrm{~nm}$ and the calculated strength of the magnetic field in the corona is shown to be a very useful instrument to solve the problem. This correlation manifests a very strong dependence both on the solar cycle phase and on the heliographic altitude. It seems reasonable that the efficiency of the mechanisms depends strongly on the relative area of low and high (including open field) loops. The models based on slow field dissipation (DC) are more 
efficient in the low-latitude zone and the wave models based on dissipation of Alfvén waves $(\mathrm{AC})$, in the high-latitude zone.

A.D. Voitsekhovska, and A.K. Yukhimuk discussed the transformation of MHD Alfvén waves and solar corona heating. One of the most interesting phenomena appearing on the Sun is the high temperature of the solar corona, which reaches $10^{6} \mathrm{~K}$ (compared with $5 \times 10^{3} \mathrm{~K}$ at the level of the photosphere). To maintain such a high temperature and compensate for radiative cooling, the constant inflow of energy is needed. The necessary energy is considered to be carried by Alfvén waves. Alfvén waves also play an important role in the heating of the solar wind and in transporting energy from the Sun to the Earth. However, MHD Alfvén waves are weakly damping and there is a problem of the transformation of energy from waves to plasma particles. Therefore, solar corona heating by Alfvén waves can be connected with transformation of long-waved Alfvén waves to short-waved kinetic Alfvén waves. Kinetic Alfvén waves are rapid damped waves and can effectively transfer energy to plasma particles. The non-linear mechanism of Alfvén wave transformation to kinetic Alfvén waves and magneto-acoustic waves in the solar coronal plasma with small plasma parameter $\beta<1$ is investigated. The parametric instability, where the Alfvén wave is a pump wave, is considered as the generation mechanism. Using a two-fluid model and Maxwell's equation, they derive a non-linear dispersion relation governing these three-wave interaction processes, the instability growth rate, and the time of instability development. Their theoretical investigations show that the decay of Alfvén waves into kinetic Alfvén and magnetoacoustic waves can cause the solar corona heating.

Paul G. Watson, and I.J.D. Craig explored the role of viscous and resistive damping of shear Alfvén waves in magnetized plasmas. Perpendicularly polarized shear waves that ride on spatially varying horizontal background fields are remarkably efficient at dissipating wave energy via phase mixing. A combination of analytical and numerical treatments confirm that solutions in simple 1D Cartesian and cylindrical geometries allow energy release rates that are only weakly dependent on the visco-resistive damping coefficients. Numerical simulations also confirm that more general 2D solutions exhibit similar behaviour. This process could provide a fast and efficient method for heating the solar corona.

The statistics of dissipation events from reduced MHD simulations was discussed by Daniel O. Gomez, and Pablo Dmitruk. Within the reduced MHD approximation, they numerically simulate the dynamics of a coronal loop driven by a stationary velocity field at the photospheric boundaries. After several photospheric turnover times, a turbulent stationary regime is reached, characterized by a broad-band power spectrum and heating rate levels compatible with the heating requirements of active region loops. The energy dissipation rate as a function of time displays a complex superposition of impulsive events, which they associate to the so-called nanoflares. A statistical analysis of these events yields a power law distribution as a function of their energies, which is consistent with those obtained for flare energy distributions reported from X-ray observations. They also study the distributions of peak dissipation rates, durations, and waiting times between events.

In order to understand the plasma processes in the solar corona, Evgeny $\mathbf{V}$. Derishev, Vitaly V. Kocharovsky, Vladimir V. Kocharovsky, and V. Yu. Martyanov studied a new class of self-consistent current sheets and filaments in collisionless plasma. They consider a continuous set of stationary current sheets and filaments in collisionless multi-component plasma found analytically using integrals of twodimensional motion of particles in the self-consistent magnetic field. In their solutions, which are relativistic in general, the magnetic energy density can be comparable to that 
of particles, and the spatial scale can be arbitrary compared to a typical gyroradius of the particles. They also consider the properties of newly found stationary solutions and their possible applications to the analysis of magnetic field configurations emerging in various astrophysical plasmas, including coronal structures, shocks and jets. The results are used for interpretation of recent observations and numerical simulations. By choosing a particular dependence of particle distribution function on the integrals of motion they are able to obtain various profiles of magnetic field and self-consistent current, including non-monotone. The obtained solutions describe a much more general class of equilibrium configurations as compared to known generalizations of Harris current sheets. On this basis, they suggest a way to describe slow dynamics and filamentation of collisionless current configurations in coronal plasma and in active galactic nuclei, $\gamma$-ray bursts, and microquasars.

Dynamics and structure of the active region NOAA 10139 on the basis of microwave observations at RT-22, SSRT, and RATAN-600 have been investigated by Yury T. Tsap, L.I. Tsvetkov, Yu.F. Yurovsky, Natalia G. Peterova, T.B. Borisevich, and B.V. Agalakov. As appears from the wavelet analysis, the observed behavior of the S-component of microwave emission is determined by elementary flare events. It has been shown that accelerated electrons can play an important role in heating the plasma of the transition region and upper chromosphere.

The Fabry-Perot interferometric study of the green coronal line during the total eclipse of 2001 from Zambia was presented by Thyagarajan Chandrasekhar, Nagarhalli M. Ashok, B.G. Ananda Rao, Jay M. Pasachoff, and Terry-Ann Suer. They reported an interesting ground-based Fabry-Perot interferometric experiment on the green coronal line at $530.3 \mathrm{~nm}$ carried out successfully during the total eclipse of 21 June 2001 from Lusaka. Unlike as in earlier experiments, a cooled CCD was used to record as many as 17 interferograms during 194 seconds of totality. The instrumental profile is well determined by a green He-Ne laser and has a FWHM of 20 pm. The Fabry-Perot was off centred with respect to the solar disk to permit wider fringe coverage of the corona. Radial scans from fringe centre of only one interferogram number over 500 and each scan has several fringes. The data base spread over 17 interferograms is huge and has been only partially analysed. The example of the interferogram is given in Fig. 5. Line-width temperatures derived from fringes analysed so far range from $2.4 \times 10^{6}$ to $3.7 \times 10^{6} \mathrm{~K}$ and many profiles are asymmetric. The data base permits a search of line width oscillations at many positions in the corona with a temporal resolution of a few seconds which has implications for wave heating of the corona.

\section{Other phenomena}

\subsection{Coronal loops}

Robert F. Wilson presented the VLA, SOHO, and RHESSI observations of magnetic interactions and particle propagation across large-scale coronal loops. Among the different types of soft X-ray and EUV coronal structures, detected by Yohkoh, SMM, and SOHO-EIT, are large-scale loops that appear to interconnect active regions across the solar equator, which are known as trans-equatorial loops. These trans-equatorial interconnecting loops are of particular interest, because they may play a role in heating and re-structuring the quiet Sun magnetic field while also being potential source regions of coronal mass ejections (CMEs). The physical mechanism that gives rise to these structures is not well understood; it is thought that the anti-parallel magnetic fields of the two active regions on opposite sites of the solar equator reconnect and form large-scale 


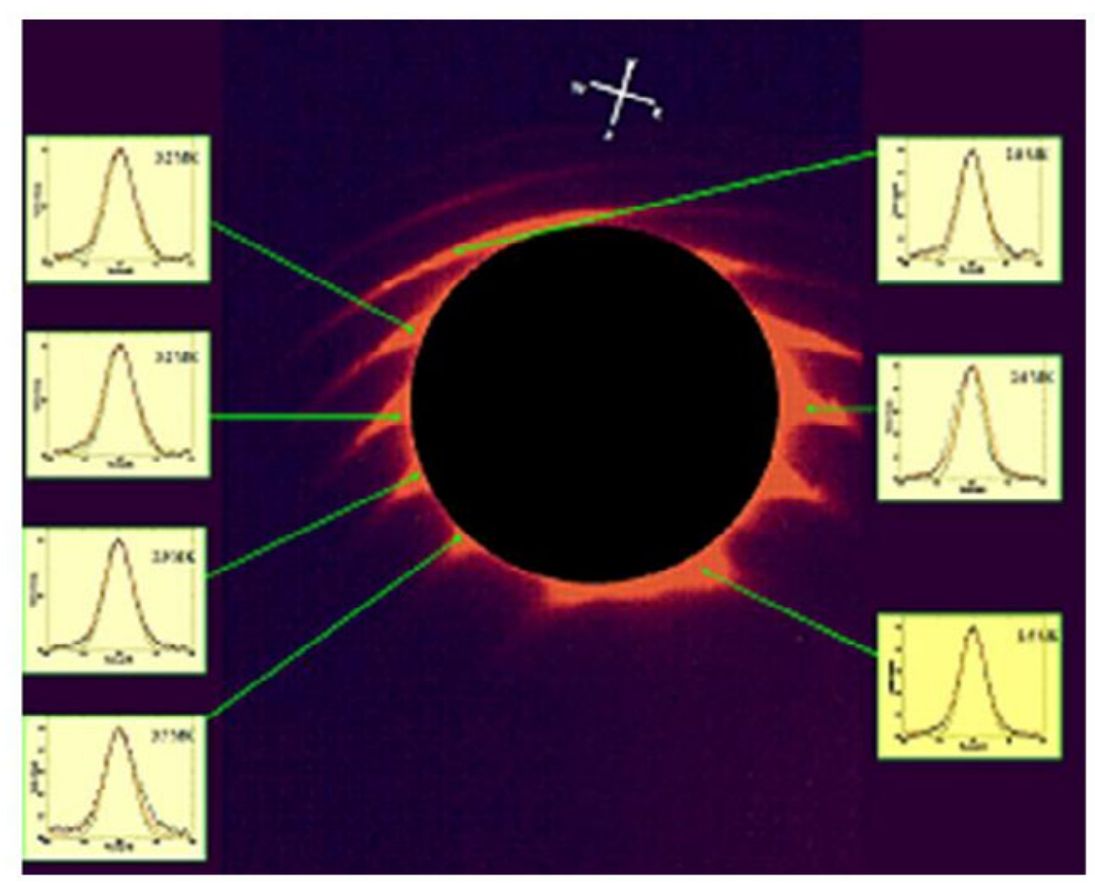

Figure 5. Coronal interferogram in $\lambda 530.3 \mathrm{~nm}$ with line profiles at selected positions.

trans-equatorial loops (TELs) in the corona, rather than coming directly from below the surface in the convective zone. In this way, the formation of these loops involves a global reconfiguration of the solar magnetic field that may play a role in heating the quiet Sun coronal plasma. In Fig. 6 is shown a series of VLA $20.7 \mathrm{~cm}$ and $91.6 \mathrm{~cm}$ wavelength snapshot maps (2 minute duration) made on July 1, 2005 that have been overlaid on SOHO-EIT images of the FexII $195 \AA$ lines taken at about the same time. The VLA $20.7 \mathrm{~cm}$ images delineate slowly-varying coronal loops that extend above and between active regions 10783 (S02E46) and 10784 (N16E57) on the solar disk. These sources have peak brightness temperatures of $T_{\mathrm{b}}=1.5 \times 10^{6} \mathrm{~K}$ and are most likely attributed to thermal bremsstrahlung or thermal gyroresonance emission. Beginning at 17:15 UT, another $20.7 \mathrm{~cm}$ source is seen to extend and separate from the western end of AR 10783 and then move northward across the solar equator with an apparent velocity of $v \simeq 55 \mathrm{~km} \mathrm{~s}^{-1}$, finally reaching AR 10784 at 18:50 UT. The path of this moving source lies along a transequatorial EUV loop connecting the two active regions. This new source appears to emerge following a gradual increase in the brightness temperature of the $20.7 \mathrm{~cm}$ source associated with AR 10783 (Fig. 6 - top) beginning 16:30 UT; it also coincides with a multi-peaked hard X-ray burst detected by RHESSI (arrow) from the eastern part of this region. The $91 \mathrm{~cm}$ emission is contained within three time-variable sources, $\mathrm{A}, \mathrm{B}$ and $\mathrm{C}$, located between the two active regions and along the TELs detected by SOHO-EIT. The long-lasting and eastern-most of the sources, source A, decreases in intensity and is replaced by a second source, B, located close to the eastern edge of AR 10783 following a second RHESSI hard X-ray burst at 17:44 UT. Finally, source B fades and is replaced by an intense burst source $\mathrm{C}$, located $\sim 1^{\prime}$ west of source $\mathrm{A}$. The appearance of the $91 \mathrm{~cm}$ 

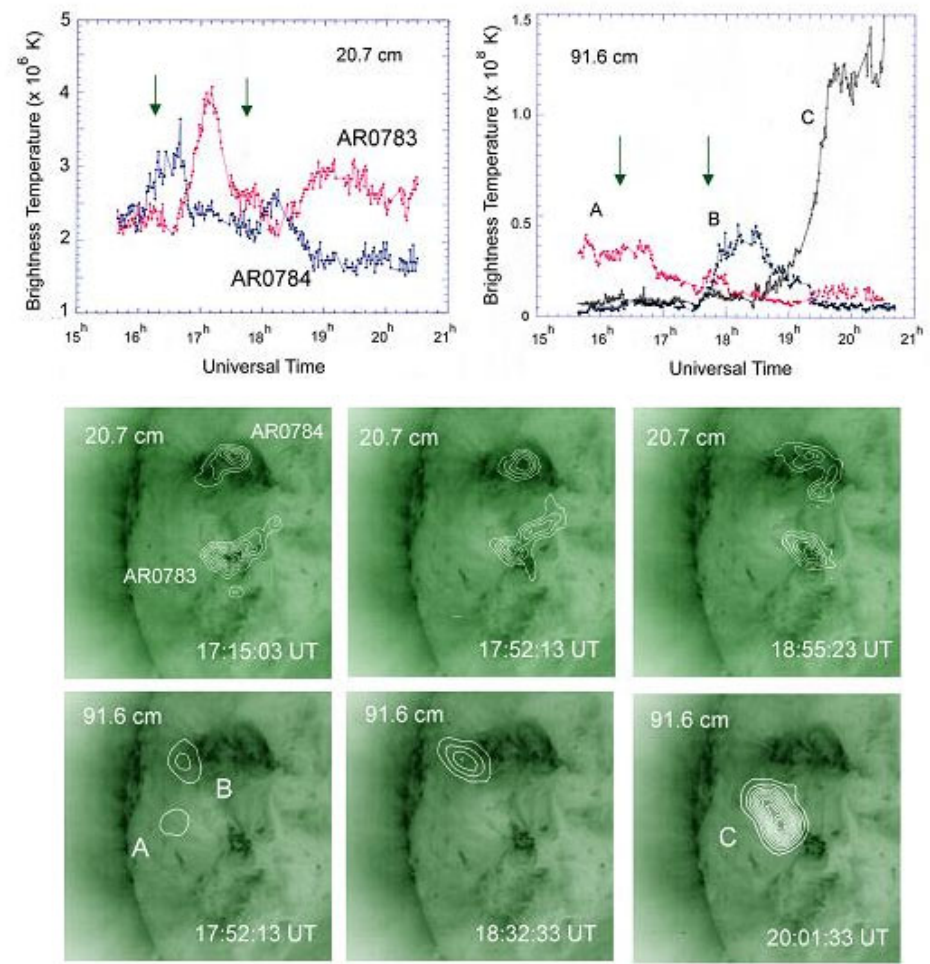

Figure 6. A plot of the peak brightness temperature (Stokes parameter I) from the sources associated with active regions AR0783 and AR0784 at $20.7 \mathrm{~cm}$ wavelength and the sources A, $\mathrm{B}$ and C labeled at $91.6 \mathrm{~cm}$ on 1 July 2005. A series of VLA snapshot maps (1 minute intervals) at $20.7 \mathrm{~cm}$ (middle row) and $91.6 \mathrm{~cm}$ (bottom row) are overlaid on SOHO-EIT Fe XII $195 \AA$ images taken at the same time. Here, the contours of the VLA maps denote levels of equal brightness temperature with an outermost contour and contour interval of $T_{\mathrm{b}}=5.0 \times 10^{5} \mathrm{~K}$. The arrows denote the onset times of hard X-ray bursts detected by RHESSI.

source $\mathrm{C}$ is also accompanied by a $50 \%$ increase in the brightness temperature of the $20.7 \mathrm{~cm}$ emission from AR 10783. Both of the $91 \mathrm{~cm}$ sources B and C are nearly $100 \%$ right-circularly polarized (source $\mathrm{A}$ is $15 \%$ left-circularly polarized).

These changes in the structure and brightness of the 20.7 and $91.6 \mathrm{~cm}$ sources that may be related to morphological changes in the large-scale EUV TELs that join the two active regions and to hard X-ray bursts in one of them. The hour-long increase in the brightness of the $20.7 \mathrm{~cm}$ emission associated with AR 10783 and the subsequent northward movement of a source across the solar equator along interconnecting loops seems to have followed a weak hard X-ray burst at 16:14 UT from AR 10783. Although there was no obvious 20.7 or $91.6 \mathrm{~cm}$ impulsive counterpart to this burst, it is possible that the magnetic reconnection event that produced the hard X-ray burst may have resulted in gradual heating of the overlying coronal loop; the TEL then acted as a conduit for hot thermal plasma which moved along the interconnecting loop to its northern footpoint. Changes in the brightness of three long-lasting, highly circularly-polarized $91 \mathrm{~cm}$ sources, possibly attributed to Type I noise storm emission, or to decimetric Type IV-like lare 

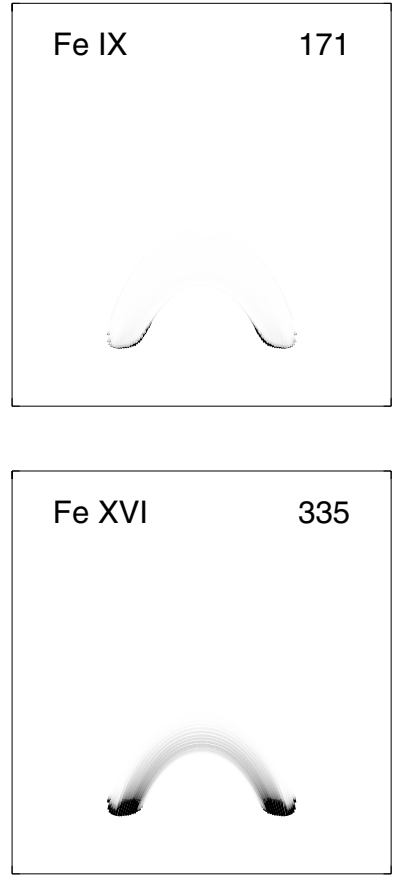
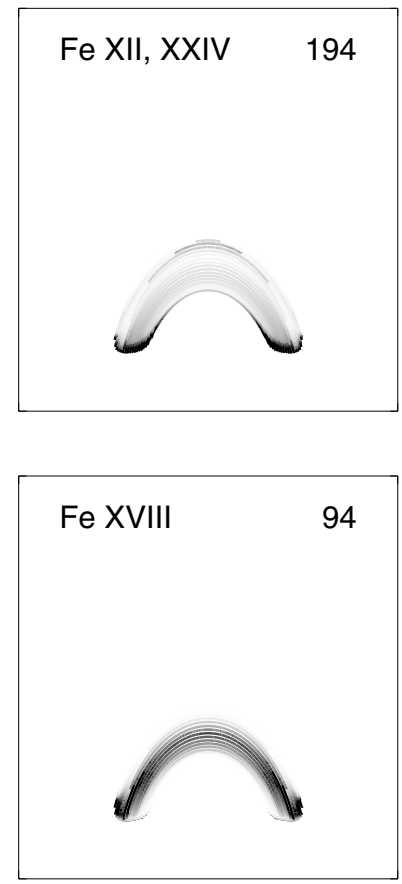
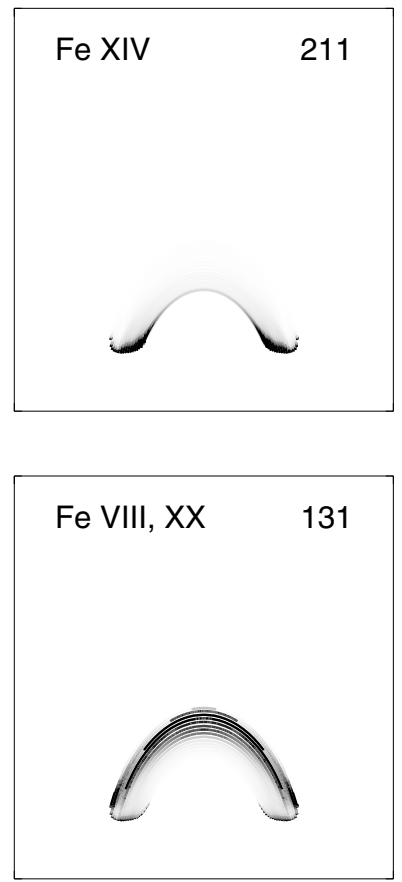

Figure 7. Simulated flare emission for the six SDO-AIA EUV wavelengths.

continuum radiation, were accompanied by continuous restructuring of the TELs along which these $91 \mathrm{~cm}$ sources appear to lie. One of these sources, B, appears at about the same time as a second hard X-ray burst from AR 10783 and may have been produced by nonthermal particles injected into the transequatorial loops during this impulsive hard X-ray event at 17:40 UT. The most intense $91 \mathrm{~cm}$ source, C, was associated with a GOES soft X-ray burst and a subsequent northward extension of the hot coronal plasma at 18:51 UT (RHESSI was in the night-time part of its orbit), and may have been triggered by continuous restructuring of the coronal magnetic environment. If the $91 \mathrm{~cm}$ sources are due to plasma emission, then the disappearance of the $91 \mathrm{~cm}$ sources $\mathrm{A}$ and $\mathrm{B}$ may be due to the fact that the emitting particles may have been displaced upward or downward in the corona by the evolving loops and therefore out of the plasma frequency level corresponding to $327 \mathrm{MHz}$, i.e., a region where the electron density is equal to $N_{\mathrm{e}}=1.3 \times 10^{9} \mathrm{~cm}^{-3}$.

Katharine K. Reeves, Harry P. Warren, and Terry G. Forbes presented a study, using a loss-of-equilibrium model for solar eruptions to calculate the thermal energy input into a system of flare loops. In this model, the flare consists of a system of reconnecting loops below a current sheet that connects the flare to an erupting flux rope. The thermal energy is calculated by assuming that all of the Poynting flux into the current sheet is thermalized. The density, temperature and velocity of the plasma in each reconnected loop are then calculated using a 1D hydrodynamic code. These parameters are coupled with the instrument response functions of various solar instruments to calculate flare emissions. They simulate spectra from the Bragg Crystal Spectrometer (BCS) on Yohkoh and find that the strong blueshifts that should be present due to the chromospheric evaporation during flare initiation are difficult to observe with BCS, but may be better observed with a more sensitive instrument. They also find that a density enhancement occurs at the top of a loop when evaporating plasma fronts in each loop leg collide 
there. This enhancement gives rise to bright loop-top intensities in simulated Transition Region and Coronal Explorer (TRACE) and Yohkoh soft X-ray Telescope (SXT) images. These loop-top features have been observed in TRACE and Yohkoh-SXT images, and are not explained by single-loop flare models. The Atmospheric Imaging Assembly (AIA) on the Solar Dynamics Observatory should be able to observe these features in detail and this model will be used to help to develop flare observing programs for SDO-AIA. A snapshot of a simulated flare viewed through the filters on the SDO-AIA telescope is shown in Fig. 7.

Hossein Safari, Sadollah Nasiri, and Yousef Sobouti discussed the effect of density stratification on a modal structure of solar coronal loops. Since the earliest identifications of the kink oscillations in coronal loops, a considerable amount of data has been analysed and possible factors causing the shift in frequency and affecting the oscillation properties of the loops were investigated by different authors. Here, analytical and numerical methods are used to understand the effects of longitudinal density variation on the coronal loops oscillations. The authors assume that the longitudinal density stratification have mathematically the same functional form at the inside and outside of the loops. They also assume that the mean density of the stratified loop is the same as that of the unstratified one, thus, for two identical loops the mass of unstratified loop is the same as of the stratified one. The density distribution along the radius of the loop is assumed to be a step function with different values for inside and outside of the loop. Equations of motion are expressed by second-order differential equations that are separable into radial and transverse components. The radial equation is solved in thin tube regime. The transverse equation is solved both by perturbation method, for small density scale heights, and numerically, otherwise. The summary of results are: $(a)$ the ratio of periods of fundamental and first overtone modes decreases markedly with increasing density scale parameter. This is in accord with the observational data of TRACE; (b) the behavior of the eigenfunctions for different longitudinal wave numbers is completely different for unstratified and stratified loops.

\subsection{Radio and microwave observations}

The radio and microwave observations are powerful tools to measure the magnetic field of solar corona. Kiyoto Shibasaki reviewed the microwave measurements of coronal magnetic field. The solar corona is filled with highly ionised plasma and magnetic field. Moving charged particles interact with magnetic field due to Lorentz force. This results in gyration motion perpendicular to the magnetic field and free motion along the magnetic field. Circularly polarized electromagnetic waves interact with gyrating electrons efficiently and the interaction depends on the sense of circular polarization (right-handed or left-handed). This is the reason why we can measure magnetic field strength through microwave observations. This process does not require complicated quantum physics but the classical treatment is enough. Hence the inversion of measured values to magnetic field strength is simpler than in the case of optical and infrared measurements. He presented the examples of distribution of magnetic field strength in the solar corona measured by the Nobeyama Radioheliograph.

Yeon-Han Kim, Yong-Jae Moon, Kyung-Seok Cho, Su-Chan Bong, and Young-Deuk Park reported on the first near-simultaneous observation of an X-ray plasma ejection (XPE), type II solar radio burst, and a coronal mass ejection (CME) on October 26, 1999. First, an XPE was observed from 21:12 UT to 21:23 UT in the Yohkoh-SXT field of view $\left(1.1\right.$ to $\left.1.4 \mathrm{R}_{\odot}\right)$. The XPE was initially accelerated and then constantly propagated with a speed of about $350 \mathrm{~km}^{-1}$. Second, a type II solar radio 
burst was observed at 21:30 UT by the Culgoora solar radio spectrograph. The burst started at the height of about $1.5 \mathrm{R}_{\odot}$, which is estimated from its starting frequency assuming the one-fold Newkirk coronal density model. From the frequency drift rate of the burst, the propagation speed is estimated to be about $400 \mathrm{~km} \mathrm{~s}^{-1}$. Third, the associated CME was observed by the Mauna Loa Mk 4 coronameter (1.2 to $\left.2.8 \mathrm{R}_{\odot}\right)$. The CME front was identified at 21:20 UT and its speed was $500 \mathrm{~km} \mathrm{~s}^{-1}$ (around $2 \mathrm{R}_{\odot}$ ) at the type II starting time. By comparing these three phenomena, they found that $(a)$ there is a remarkable difference $\left(0.4 \mathrm{R}_{\odot}\right)$ between the CME front and the XPE front at 21:23 UT, $(b)$ the type II formation height is not consistent with the CME front but with the trajectory extrapolated from the XPE front, and $(c)$ the three speeds are comparable with one another. Regarding the type II origin, their results suggest two possibilities: a coronal shock generation by the XPE front or by the CME flank.

Timothy S. Bastian reviewed radio diagnostics of magnetic fields in the solar corona. A measurement of the coronal magnetic field has been an elusive goal for many years. In recent years, however, progress has been made in developing techniques at radio and IR wavelengths to measure or constrain the magnetic field in the quiet and active corona. He discussed the radio diagnostic techniques in a variety of contexts, including the quiet corona, active regions, flares, and coronal mass ejections. Techniques that exploit emission intrinsic to the phenomena themselves - e.g., gyrosynchrotron radiation - and those which exploit external probes such as spacecraft beacons or extragalactic background sources are both discussed. Prospects for exploiting these techniques with future generations of radio instrumentation were also considered.

Stephen M. Ord presented the investigations of the solar corona with radio observations of pulsars. He proposed to examine both the magnetic field and electron content of the solar corona via measurement of the Faraday rotation and a dispersion evident in observations of background pulsar sources as they are occulted by the Sun. Utilizing a number of simultaneous lines of sight that cut different paths through the corona as the Sun rotates, it will be possible to strongly constrain the global topology of both the plasma and the magnetic field. Pulsars are periodic, broadband, polarised point sources of radio waves. In December 2006 the Sun will occult, or pass close to, a number of pulsars in the Galactic plane. He has proposed to use the Parkes radio telescope in New South Wales, Australia, to observe these pulsars throughout a ten day period at two frequencies simultaneously. The purpose is to detect any excess Faraday rotation and pulse dispersion associated with the solar corona. Although background sources have been observed through the corona successfully before, the proposed experiment differs due to the accessibility of different simultaneous lines of sight, and the ability to measure the integrated electron content and integrated magnetic field independently. He presented an outline of the intended experiments and some predictions of expected results.

Search for high-energy particle acceleration signatures in the submillimetre-visible solar flare emission spectrum was presented by Pierre Kaufmann, Antonio M. Melo, R. Marcon, A.S. Kudaka, Adolfo Marun, Pablo Pereyra, Jean-Pierre Raulin, and Hugo Levato. Recent results obtained at sub-millimeter waves indicated that key questions regarding physical mechanisms at the origin of solar flares are expected to become better understood with measurements in the far to mid-IR range. A new spectral component discovered with fluxes increasing for shorter sub-millimeter wavelengths indicates emissions by particles accelerated to very high energies. The nature of emission is not known. The observed parameters rule out a thermal interpretation, and the first emission models recently suggested assume three different mechanisms which may become comparable in importance: $(a)$ synchrotron radiation by beams of ultrarelativistic 
electrons; (b) synchrotron radiation by positrons produced by nuclear reactions arising from energetic beams interactions at dense regions close to the photosphere; and $(c)$ Langmuir waves emission from deep photosphere excited by high energy electron beams. The spectral band where observed features would be critically defined is in the far-infrared to visible range, where the terrestrial atmosphere is highly opaque. New experiments are being considered to observe solar flares from the ground in the remaining high frequency atmospheric "windows" at $670,850 \mathrm{GHz}$, and in mid- and near-infrared. Space experiments are planned for discrete frequencies between $1-20 \mathrm{THz}$. They show the first results obtained with a new setup developed to measure solar activity at $10 \mu \mathrm{m}$ (or $30 \mathrm{THz}$ ), using a camera with a focal plane array of uncooled microbolometers coupled to a celostat by an adequate optical arrangement.

\section{Concluding remarks and open questions}

\subsection{Solar databases}

In next few years, several space and ground-based observations of the solar magnetic field and associated activity will help us to understand the role of magnetic field in solar activity. Also, as Helen E. Coffey presented, the NOAA data rescue of key solar databases and digitization of historical solar images will play an important role in understanding the long term behaviour of the Sun. Over a number of years, the staff at NOAA National Geophysical Data Center (NGDC) has worked to rescue key solar databases by converting them to digital format and making them available via the World Wide Web. NOAA has had several data rescue programs where staff competed for funds to rescue important and critical historical data that are languishing in archives and at risk of being lost due to deteriorating condition, loss of any metadata or descriptive text that describe the databases, lack of interest or funding in maintaining databases, etc. The Solar-Terrestrial Physics Division at NGDC was able to obtain funds to key in some critical historical tabular databases. Recently the NOAA Climate Database Modernization Program (CDMP) funded a project to digitize historical solar images, producing a large on-line database of historical daily full disk solar images. The images include the wavelengths Ca II K, $\mathrm{H} \alpha$, and white light photographs, as well as sunspot drawings and the comprehensive drawings of a multitude of solar phenomena on one daily map (Fraunhofer maps and Wendelstein drawings). Included in the digitization are high resolution solar $\mathrm{H} \alpha \mathrm{im}$ ages taken at the Boulder Solar Observatory in 1967-1984. The scanned daily images document many phases of solar activity, from decadal variation to rotational variation and daily changes. Smaller versions are available online. Larger versions are available by request. See <http://www.ngdc.noaa.gov/stp/SOLAR/ftpsolarimages.html >.

\subsection{The questions}

The eruption or emergence of solar active regions will be the focus of observational and theoretical research of solar magnetism in the next decade. The key questions that must be addressed are:

(1) What is the height of triggering sites of explosive events? Is the magnetic field reconnection at coronal height always responsible for the destabilization of magnetic structures? It will be useful to make simultaneous observations of magnetic field at multiple heights and to compare with models to investigate this issue.

(2) How do different magnetic structures interact? 
(3) How does the field propagate beyond the chromosphere and how does it respond to photospheric flux movements?

The magnetic field in the quiet Sun has been the subject of intense study in recent years, with numerous questions waiting to be answered. Here a couple of such questions are listed as examples.

(1) What is the origin of the quiet Sun magnetic flux and how does it evolve? This question is related to the currently hotly debated issue of whether the Sun has, besides the main dynamo located near the base of the convection zone (which produces the active regions and the 11-year cycle of activity), also a turbulent dynamo within the convection zone. Of particular interest is to what extent a local dynamo acting near the surface plays a role in producing the flux present in the quiet Sun.

(2) What is the amount of quiet Sun flux and in what state is it found? This question can be subdivided into numerous sub-questions. For example: Which fraction of the magnetic flux is in the network and which fraction is in the internetwork? What is the field strength distribution in these regions? Is it equal or different? What is the exact location of the features (are all found in the intergranular lanes, or does a significant amount of flux exist also inside the upflowing bodies of granules)? Which fraction of the flux is in the form of structures that can be described by strong magnetic flux tubes, which fraction is in the form of turbulent fields?

None of these (and of many other) questions has so far found a final answer and there is a considerable controversy surrounding almost each of them. This has partly to do with the fact that outside the magnetic network, the magnetic field in the quiet Sun produces a signal in polarized light that is small and difficult to measure. New instrumentation that will start to operate in the coming months and years will play a big role in advancing our knowledge of the quiet Sun magnetic field.

\section{Acknowledgements}

We would like to acknowledge the scientific organizing committee members Drs. Takashi Sakurai, Hongqui Zhang, John M. Davis, Axel Hofmann, Sami Solanki, Tom Berger, Rob Rutten, Brigitte Schmieder, S. Ananthakrishnan, Oddbjorn Engvold, Eric Priest, Jan O. Stenflo, Donald B. Melrose and Dale Gary. Our special thanks to Dr. David F. Webb for his valuable guidance during the initial stage of preparation of this meeting. We thank the the invited speakers and the participants of the JD03 for their contributions. We also thank Dr. James A. Klimchuk for giving a summary talk at the end of this JD03.

\section{References}

Archontis, V., Moreno Insertis, F., Galsgaard, K., Hood, A., \& O’Shea, E. 2004, A\& A, 426, 1047 Aulanier, G., Démoulin, P., Schmieder, B., Fang, C., \& Tang, Y. H. 1998, A\&\&A, 183, 369

Aulanier, G., DeLuca, E. E., Antiochos, S. K., McMullen, R. A., \& Golub, L. 2000, ApJ, 540, 1126

Aulanier, G., Pariat, E., \& Démoulin, P. 2005, A\& A, 444, 961

Aulanier, G., Pariat, E., Démoulin, P., \& DeVore C. R. 2006, Solar Phys., 238, 347

Bagalá, L. G., Mandrini, C. H., Rovira, M. G., \& Démoulin, P. 2000, A\& A, 363, 779

Baranovsky E.A. 1993, Contrib. Astron. Obs. Skalnaté Pleso, 23, 107

Démoulin, P., Hénoux, J. C., \& Mandrini, C. H. 1994, A\&A, 285, 1023

Démoulin, P., Hénoux, J. C., Priest, E. R., \& Mandrini, C. H. 1996, A\&A, 308, 643

Démoulin, P., Bagalá, L. G., Mandrini, C. H., Hénoux, J. C., \& Rovira, M. G. 1997, A\&A, 325, 305

Dorch, S. B. F. 2007, AछAA, 461, 325

Fan, Y. 2004, Living Reviews in Solar Physics 1, 1 
Fletcher, L., López Fuentes, M. C., Mandrini, C. H., Schmieder, B., Démoulin, P., Mason, H. E., Young, P. R., \& Nitta, N. 2001, Solar Phys., 203, 255

Fletcher, L., Metcalf, T. R., Alexander, D., Brown, D. S., \& Ryder, L. A. 2001b, ApJ, 554, 451

Gaizauskas, V., Mandrini, C. H., Démoulin, P., Luoni, M. L., \& Rovira, M. G. 1998, A\&A, 332, 353

Galsgaard, K., Nordlund, Å. 1997, Journ. Geoph. Res., 102, 219

Hagino, M., \& Sakurai, T. 2004, Publ. Astron. Soc. Japan, 56, 831

Longcope, D. W. 2006, Living Reviews in Solar Physics 2, ... (http://www.livingreviews.org/ lrsp-2005-7)

Mandrini, C. H., Démoulin, P., Hénoux, J.-C., \& Machado, M. E. 1991, A\& A, 250, 541

Mandrini, C. H., Démoulin, P., Bagalá, L.G., van Driel-Gesztelyi, L., Hénoux, J. C., Schmieder, B., \& Rovira, M. G. 1997, Solar. Phys. 174, 229

Mandrini, C. H., Démoulin, P., Schmieder, B., Deng, Y. Y., \& Rudawy, P. 2002, A\&̛A 391, 317

Mandrini, C. H., Démoulin, P., Schmieder, B., DeLuca, E. E., Pariat, E., \& Uddin, W. 2006, Solar Phys., 238, 293

Pariat, E., Aulanier, G., Schmieder, B., Georgoulis, M. K., Rust, D. M., \& Bernasconi, P. N. 2004, A\&A, 614, 1099

Priest, E. R., \& Démoulin, P. 1995, Journ. Geoph. Res., 100, 23443

Rust, D. M., \& Kumar, A. 1994, Solar Phys., 155, 69

Titov, V. S., Priest, E. R., \& Démoulin, P. 1993, A\&A 276, 564

Titov, V. S., Hornig, G., \& Démoulin, P. 2002, Journ. Geoph. Res., 107, SSH 3, 1-13. 